\title{
Signalome-wide RNAi screen identifies GBA1 as a positive mediator of autophagic cell death
}

\author{
Santosh K Dasari', Shani Bialik', Smadar Levin-Zaidman², Vered Levin-Salomon ${ }^{1}$, Alfred H Merrill $\mathrm{Jr}^{3}$, Anthony H Futerman ${ }^{4}$ and \\ Adi Kimchi*,1
}

Activating alternative cell death pathways, including autophagic cell death, is a promising direction to overcome the apoptosis resistance observed in various cancers. Yet, whether autophagy acts as a death mechanism by over consumption of intracellular components is still controversial and remains undefined at the ultrastructural and the mechanistic levels. Here we identified conditions under which resveratrol-treated A549 lung cancer cells die by a mechanism that fulfills the previous definition of autophagic cell death. The cells displayed a strong and sustained induction of autophagic flux, cell death was prevented by knocking down autophagic genes and death occurred in the absence of apoptotic or necroptotic pathway activation. Detailed ultrastructural characterization revealed additional critical events, including a continuous increase over time in the number of autophagic vacuoles, in particular autolysosomes, occupying most of the cytoplasm at terminal stages. This was followed by loss of organelles, disruption of intracellular membranes including the swelling of perinuclear space and, occasionally, a unique type of nuclear shedding. A signalome-wide shRNA-based viability screen was applied to identify positive mediators of this type of autophagic cell death. One top hit was GBA1, the Gaucher disease-associated gene, which encodes glucocerebrosidase, an enzyme that metabolizes glucosylceramide to ceramide and glucose. Interestingly, glucocerebrosidase expression levels and activity were elevated, concomitantly with increased intracellular ceramide levels, both of which correlated in time with the appearance of the unique death characteristics. Transfection with siGBA1 attenuated the increase in glucocerebrosidase activity and the intracellular ceramide levels. Most importantly, GBA1 knockdown prevented the strong increase in LC3 lipidation, and many of the ultrastructural changes characteristic of this type of autophagic cell death, including a significant decrease in cytoplasmic area occupied by autophagic vacuoles. Together, these findings highlight the critical role of GBA1 in mediating enhanced self-consumption of intracellular components and endomembranes, leading to autophagic cell death.

Cell Death and Differentiation (2017) 24, 1288-1302; doi:10.1038/cdd.2017.80; published online 2 June 2017

Autophagy is a highly conserved process in which doublemembrane-enclosed vesicles form to consume bulk cytoplasm and organelles. It occurs in a constitutive manner to enable turn-over of long-lived proteins, removal of damaged organelles and misfolded proteins and as a defense mechanism against pathogens. ${ }^{1}$ It is induced during cell stress, nutrient deprivation or growth factor withdrawal, when its catabolic role is critical to recycle and generate cellular building blocks and energy. Thus autophagy is essential for maintenance of homeostasis and cell survival. Yet, under specific circumstances, autophagic pathways can promote cell death. The autophagic machinery and/or autophagosome can serve as platforms for caspase activation or RIP1-RIP3 complex formation, leading to apoptosis and necroptosis, respectively. ${ }^{2-8}$ Autophagy can also sensitize cells to apoptosis or necroptosis through the selective degradation of antiapoptotic or survival proteins. ${ }^{7,9-11}$ It can also drive ferroptosis, an iron-dependent form of necrosis, through autophagic degradation of the cellular iron storage protein, Ferritin. $^{12}$ In all these examples, autophagy facilitates cell death as an indirect cause.
The question remains whether the autophagic machinery by itself can lead to cell death, with no involvement of alternative cell death pathways, by overconsumption of intracellular components. This concept was suggested several decades ago, primarily based on ultrastructural observations made during insect metamorphosis, ${ }^{13}$ mammalian embryogenesis ${ }^{14}$ and mammary or prostate involution following lactation or castration. ${ }^{15}$ Later, a set of criteria was established to define autophagic cell death, whereby the death stimulus must trigger an increase in autophagic flux without activation or dependence on other programmed cell death pathways and that it can be blocked by perturbations of various autophagic proteins. ${ }^{16,17}$ Developmental autophagic cell death has been described conclusively in lower model organisms, such as Dictyostelium discoideum and Drosophila melanogaster. In Dictyostelium, autophagy is induced in response to starvation, and when combined with exposure to a second signal DIF-1, autophagic cell death results. ${ }^{18}$ In Drosophila, apoptosisindependent developmental cell death during metamorphosis mediates midgut removal by autophagy. ${ }^{19,20}$ In mammals, there have been several reports of autophagic cell death. For

${ }^{1}$ Department of Molecular Genetics, Weizmann Institute of Science, Rehovot, Israel; ${ }^{2}$ Department of Chemical Research Support, Weizmann Institute of Science, Rehovot, Israel; ${ }^{3}$ School of Biology and Petit Institute for Bioengineering and Bioscience, Georgia Institute of Technology, Atlanta, GA, USA and ${ }^{4}$ Department of Biomolecular Sciences, Weizmann Institute of Science, Rehovot, Israel

*Corresponding author: A Kimchi, Department of Molecular Genetics, Weizmann Institute of Science, Herzel Street, Rehovot 76100, Israel. Tel: +972 89342428 ; Fax: +972 8 9315938; E-mail: adi.kimchi@weizmann.ac.il

Received 03.2.17; revised 12.4.17; accepted 12.4.17; Edited by H-U Simon; published online 02.6.17 
a

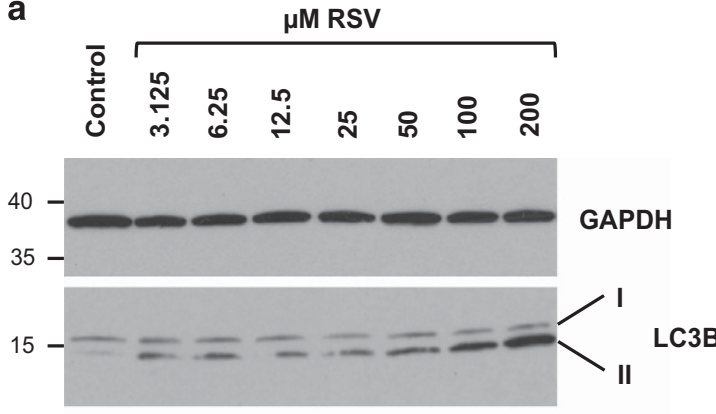

b

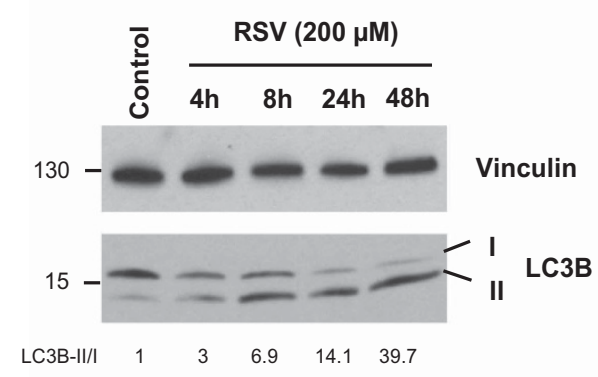

d

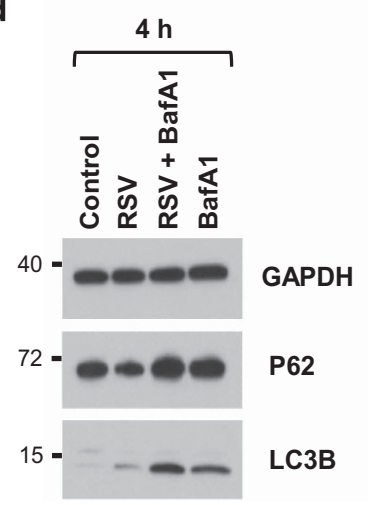

e

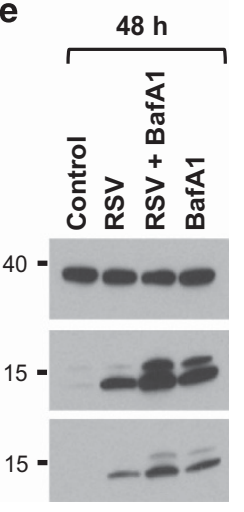

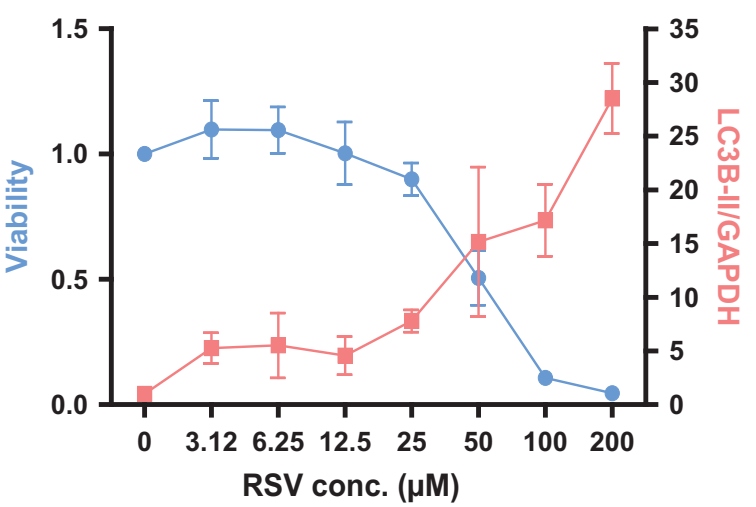

C

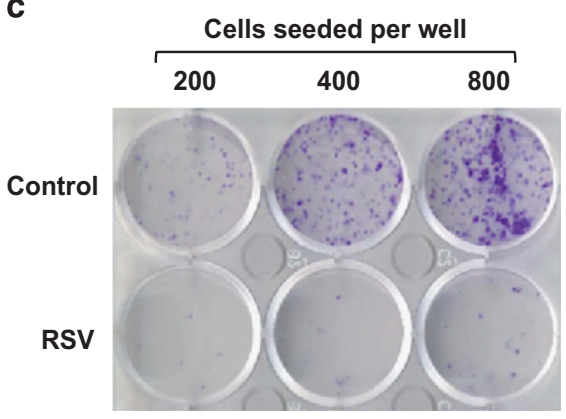

Figure 1 RSV induces autophagic cell death. (a) A549 cells were treated with different concentrations of RSV for $48 \mathrm{~h}$. Cell lysates were subjected to western blotting analysis of LC3B and GAPDH as loading control. Graph shows inverse correlation between RSV-induced LC3 lipidation (Red) and cell viability (blue), determined by CellTiter-Glo assay. Data represent mean \pm S.D. of three replicate experiments. (b) A549 cells treated with $200 \mu \mathrm{M} \mathrm{RSV}$ for 4, 8, 24 and $48 \mathrm{~h}$ were subjected to western blotting analysis with LC3B and GAPDH. (c) Representative images of clonogenic assay of A549 cells treated with $200 \mu \mathrm{M} \mathrm{RSV} \mathrm{for} 48 \mathrm{~h}$ followed by growth for additional 7-10 days. (d and e) Cells were treated with $200 \mu \mathrm{M}$ RSV for $4 \mathrm{~h}$ (d) or $48 \mathrm{~h}$ (e) in the absence and presence of 100 or $5 \mathrm{nM}$ BafA1, respectively, and western blotted for LC3B, p62 and GAPDH. (f) Representative images of A549 cells transfected with tandem RFP-GFP-LC3 and treated with or without RSV (200 $\mu \mathrm{M}, 24 \mathrm{~h})$. Scale bar, $10 \mu \mathrm{m}$

example, hypoxia/ischemia-induced hippocampal neuronal cell death in adult mice was dependent on autophagy gene ATG7 and independent of apoptosis. ${ }^{21}$ Another autophagic cell death pathway, termed autosis, was induced by a cellpermeable peptide-activating Beclin- $1^{22}$ and was likewise observed in pathophysiological settings, such as starvation in vitro and hypoxia-ischemia and aneroxia-nervosa in vivo. ${ }^{22,23}$ Cell death by autosis, which was independent of apoptosis or necroptosis, was mediated by $\mathrm{Na}^{+} / \mathrm{K}^{+}$-ATPase, suggesting that changes in ion transport and/or cellular osmolarity caused cell death. ${ }^{22}$

Understanding what converts autophagy from a protective mechanism to a lethal one is currently limited by the lack of morphological and molecular characterization of the process. Questions such as whether autophagic cell death results from selective degradation of factors necessary for cell survival or from overactivation of non-selective autophagy leading to irreversible disintegration of the cell have not been resolved. Additionally, it is not known whether disabling regulatory processes that usually serve to limit autophagic flux in stressed cells is sufficient to lead to cell death or whether additional molecular pathways need to be turned on simultaneously in analogy to the DIF-1 second signal in Dictyostelium. Identifying specific molecular mechanisms is an essential step in substantiating the existence of autophagic cell death. 
To address these issues and to identify positive regulators/ executioners of autophagic cell death in a systematic manner, we conducted a functional RNAi-based positive screen for identifying genes that are indispensable for lethal autophagy in a cell system that fulfills the strict definition of autophagic cell death and displays ultrastructural changes that result in cellular dismantling. The screen led to the identification of the GBA1 gene, encoding glucocerebrosidase (GCase). GCase protein and enzymatic activity are elevated at late stages during autophagic cell death, resulting in upregulation of intracellular ceramide levels. Molecular and morphological assessment of GBA1 knockdown (KD) cells implied that the upregulation of GCase is critical for the enhanced selfconsumption of intracellular components, leading to endomembrane catastrophe and cell death.

\section{Results}

Resveratrol (RSV) induces autophagic cell death. The model cell system chosen to dissect molecular aspects of autophagic cell death utilized RSV treatment of A549 human lung carcinoma cells, as it met the strict definition of autophagic cell death. RSV induced a dose-dependent induction of LC3 lipidation in A549 cells, indicative of autophagy activation (Figure 1a). The increase in LC3 lipidation inversely correlated with cell viability, which sharply declined at high RSV concentrations (Figure 1a). There was a continuous time-dependent increase in LC3 lipidation at lethal dosage (200 $\mu \mathrm{M} R \mathrm{RV})$, reaching maximal levels by $48 \mathrm{~h}$ (Figure 1b). A clonogenic survival assay demonstrated that $48 \mathrm{~h}$ treatment with $200 \mu \mathrm{M}$ RSV treatment significantly suppressed the colony-forming activity of A549 cells (Figure 1c). Notably, LC3 lipidation levels further increased upon simultaneous treatment with RSV and the lysosomal inhibitor Bafilomycin A1 (BafA1) for $4 \mathrm{~h}$, compared with either agent alone, indicating that the enhanced LC3 lipidation was due to induced autophagosome biogenesis, and not a block in autophagosome clearance (Figure 1d). In addition, the autophagic receptor p62 was degraded upon RSV treatment, which was blocked by BafA1 (Figure 1d). Increased autophagic flux was maintained up to $48 \mathrm{~h}$ (Figure 1e), the time point when dying cells were observed (see below). Autophagic flux was also monitored by transfection with mRFP-EGFP-LC3. RSV-treated cells showed a high density of red puncta, representing autolysosomes, wherein the GFP signal is quenched by the acidic environment (Figure 1f). Thus RSV activates autophagy flux and cell death in A549 cells.

Notably, there was no evidence for activation of apoptosis and/or necroptosis in RSV-treated A549 cells. The cells did not show any apoptotic characteristics, such as nuclear condensation or fragmentation (Supplementary Figure S1a). In addition, caspase-9, caspase-8 and caspase-3 were not cleaved/activated upon RSV treatment, in contrast to the positive control, TRAIL and cycloheximide treatment (Figure 2a). Likewise, there were no detectable signs of enhanced MLKL phosphorylation on T357/S358, a marker of necroptosis and RIPK3 activity, unlike in the positive control, HT-29 cells treated with TNFa, the IAP antagonist BV6 and z-VAD ${ }^{24}$ (Figure 2b). Furthermore, neither the pan-caspase inhibitor Q-VD, nor the RIPK1 inhibitor Necrostatin-1 and MLKL-specific inhibitor necrosulphonamide (NSA) rescued RSV-induced cell death (Figure 2c). Examination of ROS levels using cell-permeable $\mathrm{H}_{2}$ DCFDA indicated an increase following $48 \mathrm{~h}$ RSV treatment (Supplementary Figure S1b). Simultaneous treatment with RSV and ROS scavenger $\mathrm{N}$-acetyl cysteine failed to rescue cell viability (Supplementary Figure S1c), indicating that ROS signaling is not a rate-limiting event in RSV-induced cell death.

Significantly, the KD of essential autophagy genes ATG7 and ATG12 by siRNA decreased RSV-induced LC3 lipidation, as expected, and most importantly, increased cell viability (Figure 2d). Similarly, death responses were alleviated by the KD of $A T G 4 B$ and MAP1LC3B, performed as part of a siRNA screen (see Figures $5 \mathrm{~b}$ and $\mathrm{c}$ below). Overall, these data indicate that RSV-induced cell death is distinct from apoptosis and necroptosis and instead requires the functional basic machinery of autophagy and active autophagic flux, thus it is classified as autophagic cell death.

Interestingly, LC3 lipidation and cell death induced by RSV were not prevented by depletion of upstream regulators Beclin-1 and ULK1 (Figure 2e), suggesting that RSV activates non-canonical autophagy. Recent reports have identified the Golgi as a main membrane source for non-canonical autophagy. ${ }^{25,26}$ Accordingly, autophagy-dependent degradation of p62 by RSV was partially blocked by the Golgi destabilizing agent, Brefeldin A (Figure 2f). Co-transfection of A549 cells with GFP-LC3 and the Golgi marker mCherry-

\footnotetext{
Figure 2 RSV induces apoptosis- and necroptosis-independent cell death. (a and b) Immunoblot analysis of apoptotic (a) and necroptotic (b) markers in RSV (200 $\mu$ M; $48 \mathrm{~h}$ ) treated A549 cells. A549 cells treated with $100 \mathrm{ng} / \mathrm{ml}$ TRAlL and $20 \mu \mathrm{g} / \mathrm{ml}$ cycloheximide (CHX) for $2 \mathrm{~h}$ were used as a positive control for apoptosis, and HT29 cells treated with $100 \mathrm{ng} / \mathrm{ml} \mathrm{TNF}, 1 \mu \mathrm{M}$ IAP antgonist BV6 and $20 \mu \mathrm{M}$ Z-VAD (TBZ) for $8 \mathrm{~h}$ served as a positive control for necroptosis. Cleaved caspase-3 blot was reprobed with caspase-8 antibody. Phosphorylated MLKL (pMLKL) blot was reprobed with total MLKL antibody. (c) A549 cells treated with RSV (200 $\mu \mathrm{M} ; 48 \mathrm{~h})$ in the presence and absence of pancaspase inhibitor Q-VD $(10 \mu \mathrm{M})$, MLKL inhibitor NSA $(10 \mu \mathrm{M})$ or RIPK1 inhibitor necrostatin-1 (Nec-1, $10 \mu \mathrm{M})$ were assessed for cell viability using CellTiter-Glo assay. Data represents mean \pm S.D. of three replicate experiments. (d) A549 cells transfected with either ATG7 or ATG12 siRNAs were treated with RSV (200 $\mu \mathrm{M} ; 48 \mathrm{~h})$ and subjected to western blotting analysis for LC3B lipidation. Cell viability was assessed using CellTiter-Glo assay and represented as fold-change in RSV-treated cells compared with untreated cells. Data represents mean \pm S.D. of four replicate experiments; statistical significance was assessed using one-way analysis of variance (ANOVA) followed by Tukey's post hoc test, ${ }^{* \star} P<0.01 ;{ }^{* \star} P<0.001$. (e) A549 cells transfected with either Beclin 1 or ULK-1 siRNAs were treated with RSV $(200 \mu \mathrm{M} ; 48 \mathrm{~h})$ and subjected to western blotting analysis for LC3B lipidation. Cell viability was assessed using CellTiter-Glo and represented as a ratio of ATP levels in RSV-treated cells versus untreated cells. Data represent mean \pm S.D. of three independent experiments, statistical significance was assessed using one-way ANOVA followed by Tukey's post hoc test; NS: non-significant. (f) A549 cells were treated with RSV (200 $\mu \mathrm{M} ; 8 \mathrm{~h})$ in the absence or presence of Brefeldin A and subjected to western blotting. Shown is a representative immunoblot of p62 degradation. (g) GFP-LC3 and mCherry-GalT expressing A549 cells treated with RSV $(200 \mu \mathrm{M} ; 24 \mathrm{~h})$ were processed to determine colocalization between GFP-LC3-positive and mCherry-GalT-positive puncta. Arrows point to regions of Golgi showing colocalization with GFP-LC3 puncta in RSV-treated cells
} 
GalT, followed by treatment with RSV for $24 \mathrm{~h}$, showed some overlap between mCherry-GalT and GFP-LC3, supporting the possibility that the Golgi serves as a source for autophagosome biogenesis in RSV-induced autophagy (Figure $2 \mathrm{~g}$ ).

RSV leads to enhanced autolysosome formation. Ultrastructural examination by TEM of RSV-treated A549 cells revealed dramatic changes reflecting various temporal stages of autophagic cell death. Although control A549 cells showed normal ultrastructure, mitochondria, ER and Golgi with few autophagic vacuoles (AVs) (Figure 3a), RSV-treated cells $(48 \mathrm{~h})$ contained numerous AVs, including doublemembrane-bound structures (autophagosomes) and singlemembrane-bound structures with electron-dense material in the lumen (autolysosomes) (Figures $3 b$ and $c$ and Supplementary Figure S2d). Examination of the AV content

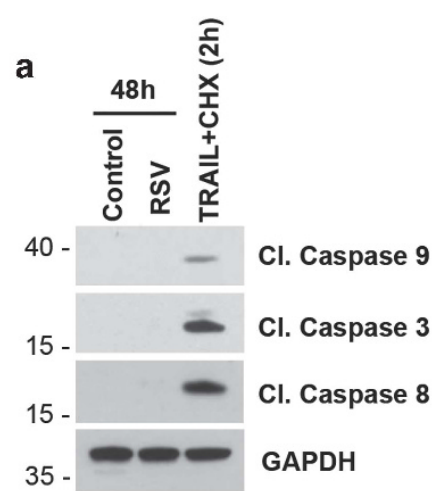

d

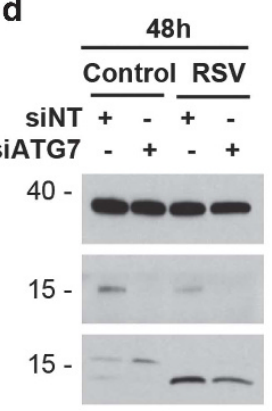

GAPDH

ATG7

LC3B

e $48 \mathrm{~h}$ Control RSV siNT + - + siBeclin-1 - + +

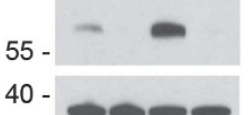

Beclin-1

40 $00-$ 15

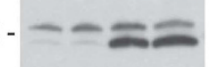

GAPDH

LC3B

$\mathbf{f}$
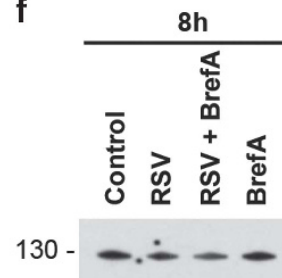

72

55

p62/Vinculin $1 \quad 0.3 \quad 0.8 \quad 1.1$

Vinculin

p62 b

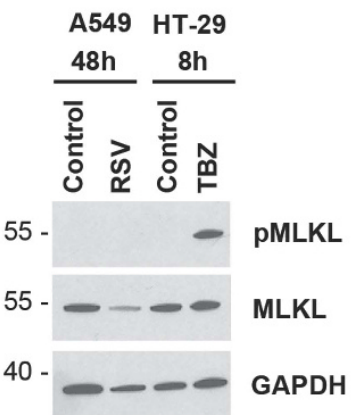

$48 \mathrm{~h}$
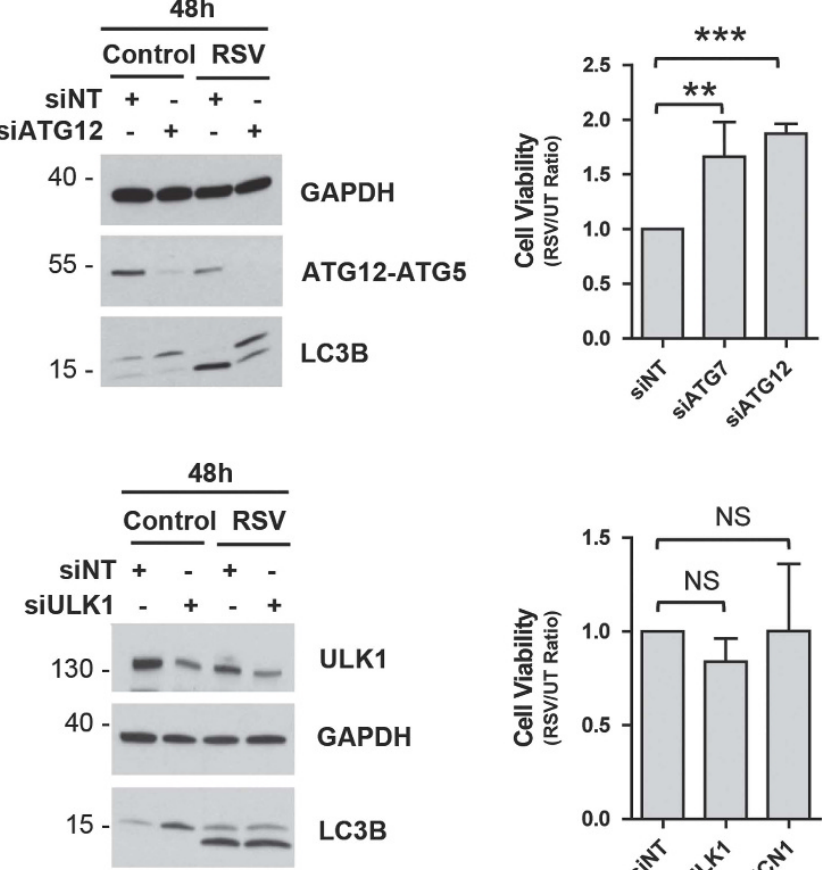

ULK1

GAPDH

LC3B

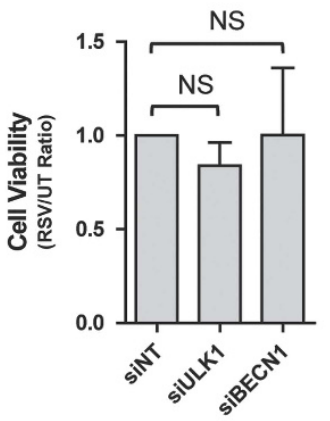

g
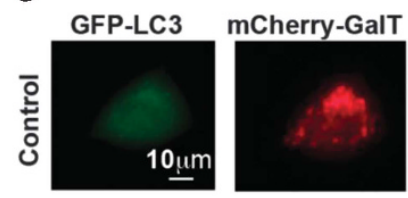

Merge
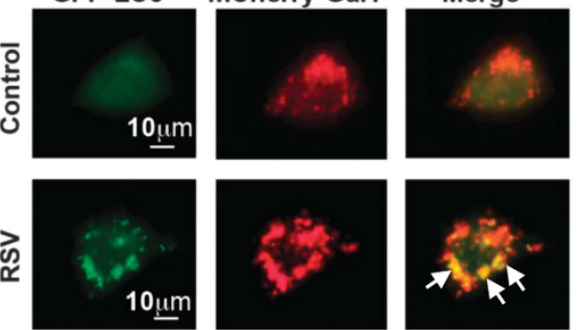
revealed degraded mitochondria, intracellular membranes and cytoplasmic material (Figures 3b, c and g), indicating that bulk macroautophagy occurred in these cells. Quantification of the AVs indicated a gradual increase in the average number of AVs per cell and an increase in the percentage of AV versus cytoplasmic area per cell from 8 to $48 \mathrm{~h}$ (Figures $3 e$ and $f$ ). Most of the cells treated with RSV for $8 \mathrm{~h}$ displayed a moderate increase in the number of AVs and swollen Golgi, yet retained normal ER, mitochondria and nuclear morphology (Supplementary Figure S2a). At $24 \mathrm{~h}$, in addition to the continued increase in the number of AVs, several hallmarks of cellular stress were prominent, including empty vacuoles, abnormal mitochondria, swollen Golgi and ER and enlarged perinuclear space, which in most cells, was uniform around the nucleus (Supplementary Figure S2b). Notably, many of the AVs were found in close proximity to the Golgi (Supplementary Figure S2c). At $48 \mathrm{~h}$, the number of AVs and percentage of AV area versus cytoplasmic area continued to increase (Figures $3 b, c$, $e$ and $f$ and Supplementary Figure S2d), until most of the organelles and cytosol were consumed by autophagy (Supplementary Figure S3b), leaving empty vacuoles. The perinuclear space was further swollen, creating a large separation between the inner and outer nuclear membranes (Figures $3 c$ and d, and Supplementary Figure S2d). Notably, and in contrast to apoptotic and necrotic cells, RSV-treated cells were strongly adherent, even at the final stages of death. The plasma membrane remained intact in most of the cells, as documented by the lack of PI staining up to $48 \mathrm{~h}$ (not shown). Occasionally nuclear shedding was detected, leaving behind the ghost of dead cells attached to the plate (Figure 3h). This was also visible under light microscopy without cell fixation (Figure 3i) and was unique to RSV-induced autophagic cell death.

Next, RSV-treated cells were stained with Lysotracker Red DND-99, which emits red fluorescence within acidic vacuoles. Lysotracker Red staining increased in RSV-treated cells and was dispersed throughout the cytoplasm, in contrast to control cells, which displayed perinuclear clustering (Figures $4 a$ and b). BafA1-treated cells did not show positive Lysotracker Red staining (Supplementary Figure S3a). The redistribution of acidic compartments in RSV-treated cells is consistent with the increased AV number. To determine whether the empty vacuoles were late-stage autolysosomes in which cellular content was degraded (Supplementary Figure S3b), we performed immunogold labeling of A549 cells transfected with LAMP1-GFP or RFP-GFP-LC3 and treated with RSV for $24 \mathrm{~h}$. Both empty vacuoles and single membrane vacuoles containing cargo were positive for LAMP1 (Figure 4c and Supplementary Figure S3c) and LC3 (Figure 4d and Supplementary Figure S3d), supporting the autolysosomal nature of these vacuoles. Together, the ultrastructural morphology and the increased lysosomal staining that corresponds to abundant autolysosomes are consistent with enhanced autophagic degradation, supporting the hypothesis that excessive consumption/hyperautophagy led to cell death.

RNAi screen to identify novel modulators of autophagic cell death. To identify genes and pathways involved in RSVinduced autophagic cell death, an shRNA-based survival screen was performed using Cellecta's Lentiviral shRNA Library Module 1 targeting 5043 signaling pathwayassociated genes ( $\sim 5$ shRNAs/gene). The screen was based on the hypothesis that cells expressing shRNAs targeting genes necessary for autophagic cell death pathways would show a reproducible survival advantage to the death signal. These shRNAs would be overrepresented when compared with their respective levels before treatment, upon calculation by shRNA barcode analysis (Supplementary Figure S4a). A549 cells were transduced with the library and then treated with $200 \mu \mathrm{M}$ RSV for 4 days, followed by a 4 day recovery. The distribution of all shRNAs in these samples (T8), compared with transduced but untreated cells (T0), was analyzed using deep sequencing. Data obtained for all shRNAs was normalized to the mean of the shRNAs targeting luciferase, which served as an internal control. Data from biological duplicates showed a good degree of correlation (Supplementary Figure S4b). The fold-change ratios of the normalized read counts for all shRNAs was calculated by dividing the RSV-treated values (T8) with the corresponding reference sample values (TO). The derived fold-change ratios were log transformed and plotted. From the $10 \%$ highest scoring shRNAs, hits were restricted to 55 candidate genes (Figure $5 \mathrm{a}$ and Supplementary Table S1), based on the following criteria: (1) enrichment of three or more independent shRNAs per gene, (2) fold change $\geq 1.5$, (3) $P \leq 0.05$, and (4) FDR $<0.1$ (Supplementary Figure S4a). Notably, ATG4B, a key autophagy gene that modulates lipidation of LC3B and other ATG8 homologues, was among the top hits. Gene Ontology analysis of the top 55 genes indicated small-molecule metabolism, negative regulation of glucuronosyltransferase activity and lipid metabolism as significantly enriched terms (Supplementary Figure S4c). The genes involved in lipid metabolism, transport and binding are particularly interesting considering the cellular membrane changes that accompanied RSV-induced autophagy. In addition, KEGG pathway analysis showed enrichment of genes associated with metabolic pathways, pathways in cancer and the lysosome (Supplementary Figure S4c). Included in the latter group were CTSE and GBA1, encoding lysosomal enzymes cathepsin E and GCase, respectively. The emergence of UGT1A9, encoding UDPGlucuronosyltransferase $1 \mathrm{~A} 9$, might be linked to the finding that this enzyme can glucuronidate RSV and modify its bioavailability and efficacy. ${ }^{27,28}$ Transferrin (TF), an ironbinding transport protein that is involved in ferroptosis, was also identified among the positive hits, suggesting that changes in cellular iron content may be critical for this form of autophagic cell death. ${ }^{29,30}$

To further prioritize the hits, a sum of ranked standardization scores algorithm ${ }^{31}$ was applied and the top 24 hits were selected for further validation in a siRNA-based secondary screen (Figure 5b and Supplementary Table S2). A549 cells were reverse transfected with a custom siRNA library targeting these genes and with additional controls. siRNA against the autophagy gene MAP1LC3B was included as a positive reference. Cell viability was assessed after $48 \mathrm{~h}$ RSV treatment using the CellTiter Glo assay. Data obtained from biological triplicates showed a high degree of correlation (Supplementary Figure S4d). This short-term assay, 

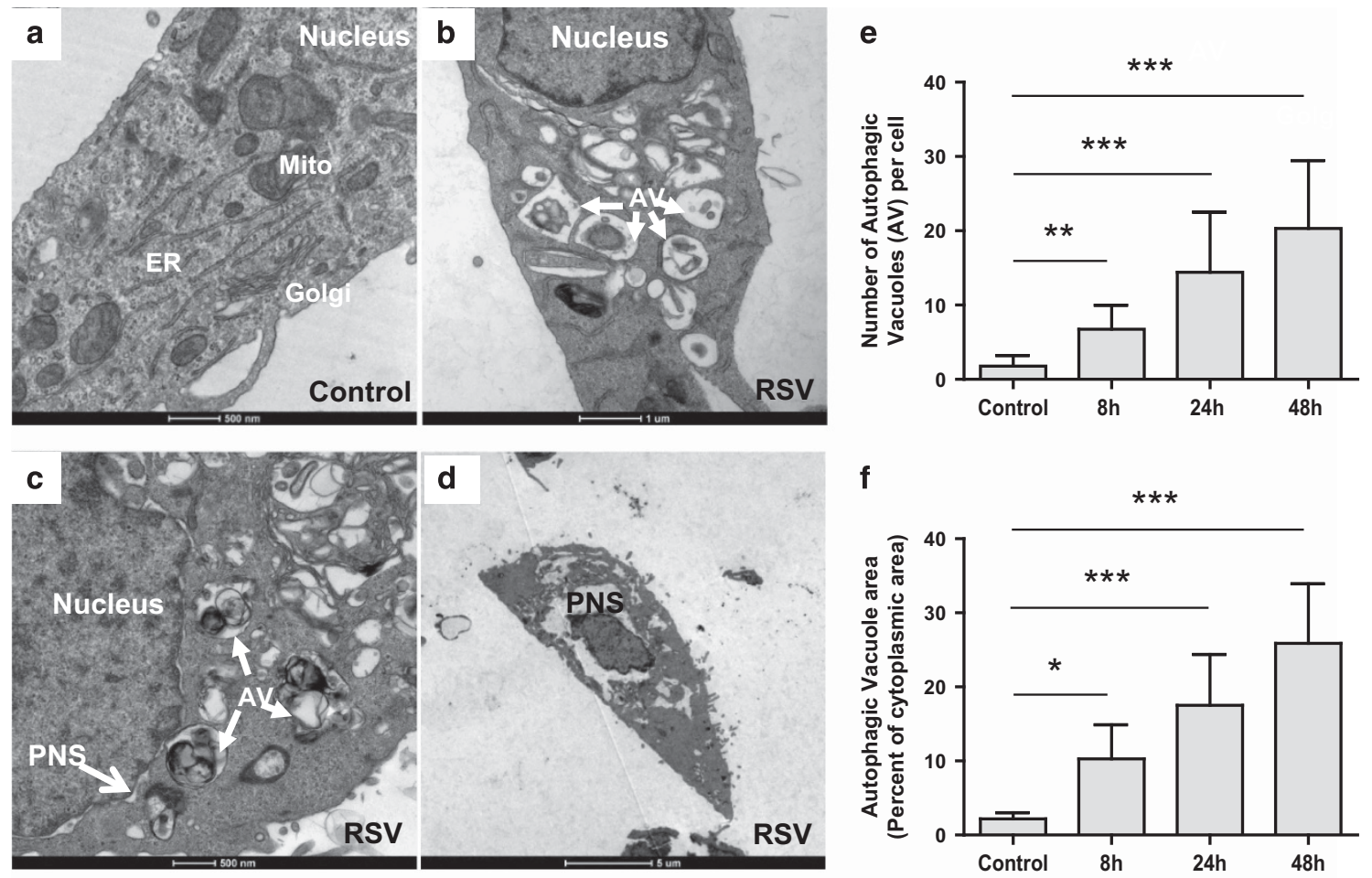

g

Macroautophagy

Cytoplasm and membrane sequestration
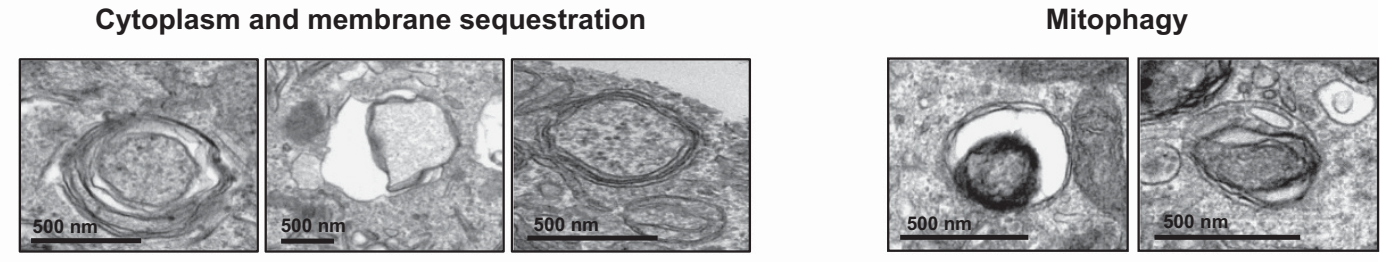

$\mathbf{h}$
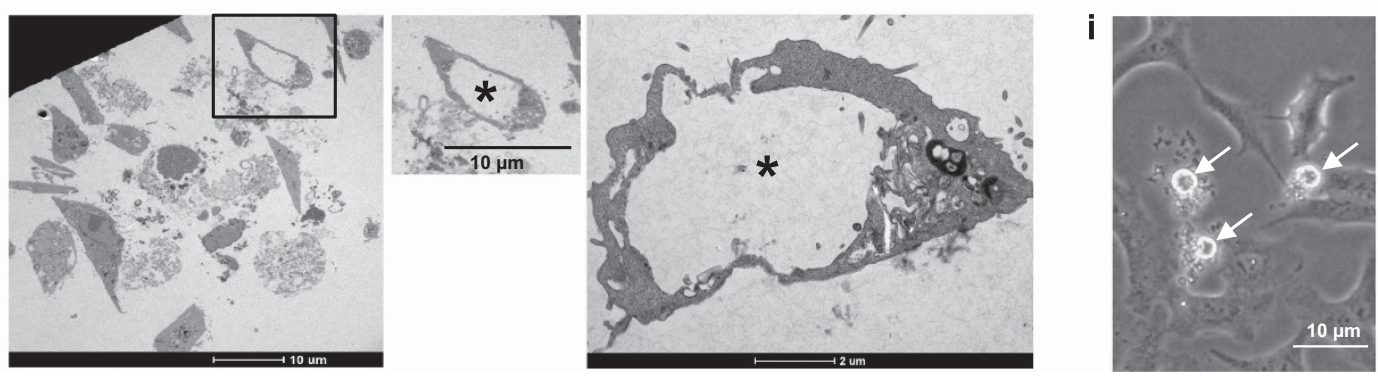

Figure 3 Ultrastructural features of RSV-induced autophagic cell death. A549 cells were treated with $200 \mu \mathrm{M}$ RSV for $48 \mathrm{~h}$ and viewed by TEM. (a) Control cells with normal ultrastructure. (b-d) Representative RSV-treated cells showing increased number of AVs, defective mitochondria (b) and increased perinuclear space (PNS, $\mathbf{c}$ and $\mathbf{d}$ ). (e and f) Quantitation of AV number per cell ( $n=30-50$ cells) and the percentage of cytoplasmic area covered by AVs ( $n=10$ cells) for control and RSV-treated cells (200 $\mu$ M for 8,24 and $48 \mathrm{~h})$. Data represent mean \pm S.D. of three replicate experiments; statistical significance was assessed using one-way analysis of variance followed by Tukey's post hoc test, ${ }^{\star} P<0.05$; ${ }^{* \star} P<0.01$; ${ }^{\star \star \star} P<0.001$. (g) Representative images of AVs harboring partially degraded mitochondria, cytoplasmic material and subcellular membranes. (h) Representative images of enucleated cells observed in RSV-induced autophagic cell death. Asterisk denotes cellular area previously occupied by nucleus. (i) Representative image of floating nuclei (indicated by arrows) surrounded by cellular debris attached to the plate

which was more stringent than the initial long-term positive selection, narrowed the list down to eight genes whose KD conferred $>50 \%$ increase in cell viability (i.e., RSV/Untreated ratio $\geq 1.5$ ) (Figure $5 b$ ). KD of four genes from this list, specifically TF, UGT1A9, ATG4B and GBA1, rescued cell viability to an extent comparable to the KD of MAP1LC3B (Figure 5c).
GCase regulates autophagic cell death. GCase, which is encoded by GBA1, metabolizes glucosylceramide (GlcCer) to ceramide and glucose within the lysosome. Lysosomal ceramide is further converted to sphingosine by acid ceramidase, which then exits the lysosome and can be reconverted to ceramide by ceramide synthases on the ER. ${ }^{32}$ Consistent with GBA1's status as a top functional hit, GCase 

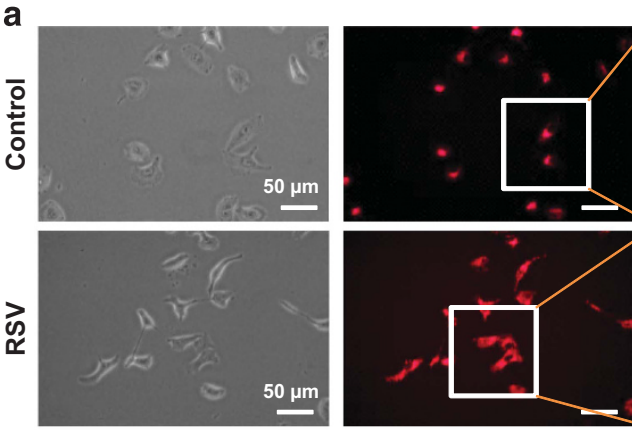

C

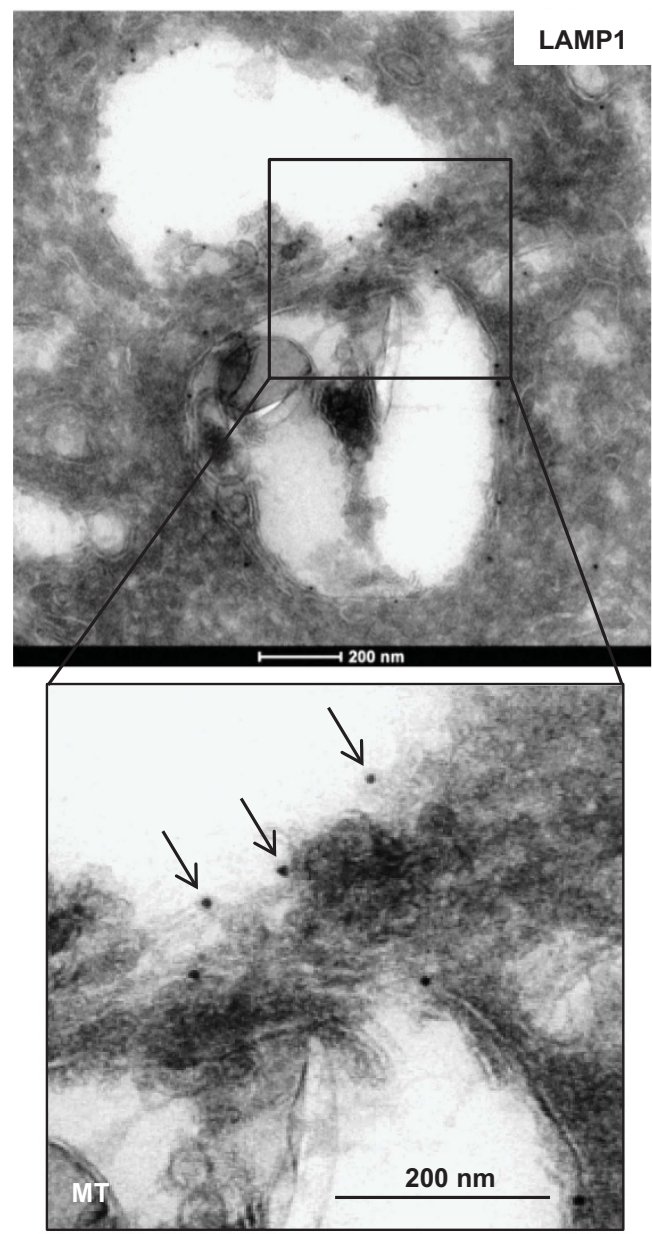

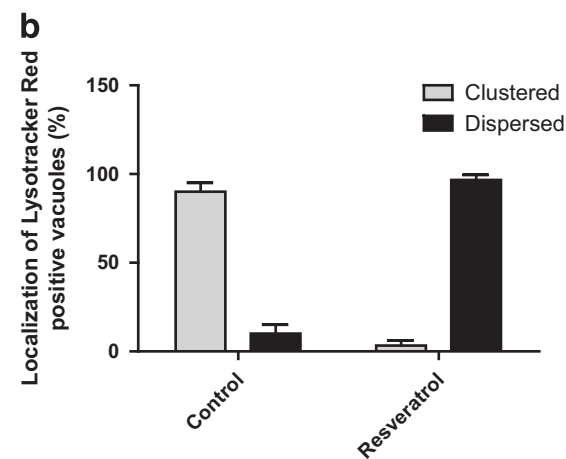

d

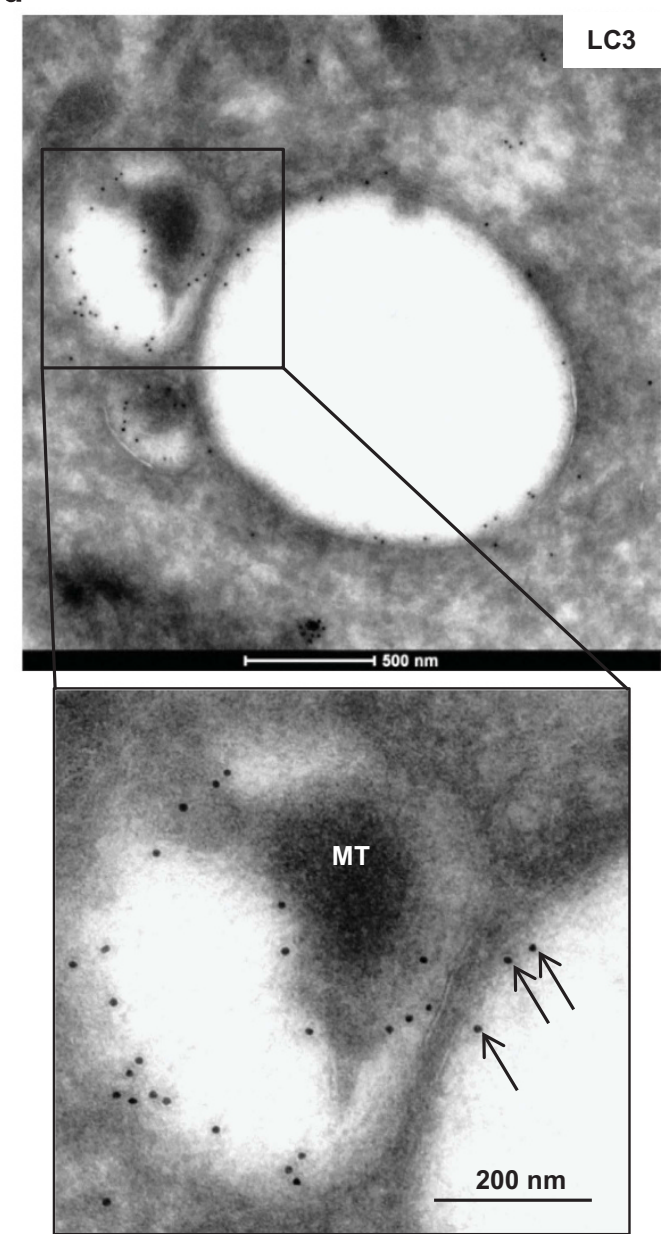

Figure 4 RSV enhances autolysosomal numbers. (a) Control and RSV (200 $\mu \mathrm{M}, 24 \mathrm{~h})$ treated A549 cells were stained with Lysotracker Red and visualized by light (left panels) and fluorescent microscopy. Panel on right is magnification of boxed region in the middle panel. (b) Quantitative representation of percentage of cells showing perinuclear clustering or dispersed Lysotracker Red staining in the absence or presence of RSV for $24 \mathrm{~h}$. Values are expressed as mean \pm S.D. of three experiments, with $>200$ cells counted per experiment. (c and d) Immunogold labeling for LAMP1-GFP (c) and mRFP-EGFP-LC3 (d) in $24 \mathrm{~h} \mathrm{RSV-treated} \mathrm{A549} \mathrm{cells.} \mathrm{Panels} \mathrm{shown} \mathrm{on} \mathrm{bottom} \mathrm{are}$ magnifications of boxed areas shown on top. Arrows indicate gold particles associated with the membrane of empty vacuoles. MT, mitochondria

protein levels were elevated $\sim 3$-fold in the presence of RSV under autophagic cell death-inducing conditions $(200 \mu \mathrm{M}$ RSV; $48 \mathrm{~h}$ ) (Figure 6a). This elevation occurred between 24 and $48 \mathrm{~h}$ when the number of AVs was maximal (Supplementary Figure S5a, compare to Figures $3 e$ and f). Similar increases in GCase were observed in additional cell lines that induced autophagy in response to RSV, including
HeLa, SH-SY5Y, HEK293 and PC3 (Supplementary Figure S5b). Importantly, the dose-response curve of GCase induction by RSV in A549 cells matched the observed reduction in cell viability (Supplementary Figure S5c, compare to Figure 1a). Interestingly, GCase levels did not change in A549 cells starved of nutrients, which undergo canonical survival autophagy (Supplementary Figure S5d). Consistent 

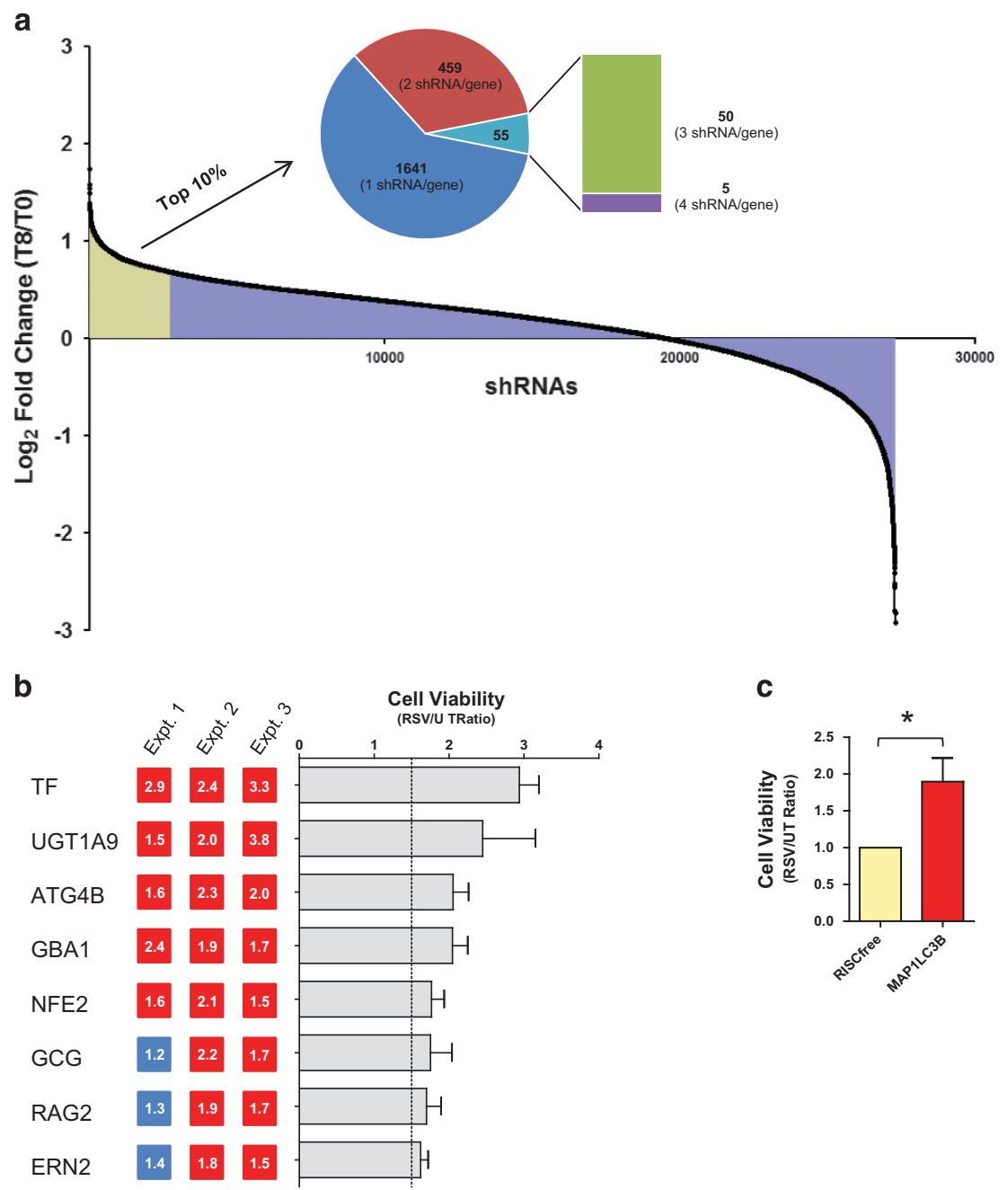

Figure 5 RNAi-mediated screen for modulators of autophagic cell death. (a) Graphical representation of pooled Lentiviral shRNA-based primary screen results, ranked from most enriched to most depleted. The $y$ axis represents the average log2 fold-change (T8/T0) value from two replicate screens. Pie chart represents top $10 \%$ significant hits obtained from the pooled shRNA screen. In total, 55 genes with $\geq 3$ shRNA hits overrepresented in T8 sample compared with T0 sample were taken for further analysis. (b) Custom cherry-picked siRNA library plates (96 well) prepared from high-ranking hits were reverse transfected and $48 \mathrm{~h}$ later, treated with RSV for another $48 \mathrm{~h}$. Cell viability was measured with CellTiter-Glo, and the fold changes were normalized to RISC-free siRNA-transfected wells. Shown are the KDs resulting in at least $50 \%$ cell death rescue (demarcated by dotted line). Values are the mean \pm S.E.M. of three independent experiments; results of individual experiments are shown at left. KD conditions showing $>50 \%$ rescue are indicated in red, whereas siRNA KD showing $<50 \%$ rescue are indicated in blue. (c) Graph comparing extent rescue in cell viability of the positive control MAP1LC3B to negative control. Data represent fold-change as mean \pm S.E.M. of three replicate experiments. Statistical significance determined by two-tailed unpaired Student's $t$-test, ${ }^{*} P<0.05$

with the changes in protein expression, GCase enzyme activity was also elevated by RSV at $48 \mathrm{~h}$ (Figure 6b). Analysis of the sphingolipid profile by mass spectrometry revealed that prolonged exposure ( $48 \mathrm{~h}$ ) to RSV resulted in an overall increase in all sphingolipids examined, including ceramide, sphingosine and sphingosine-1-phosphate (S1P) (Figures 6c, e and f). The ceramide species that increased included a variety of acyl chains (Supplementary Figure S5e). No significant changes in ceramide levels were detected at $24 \mathrm{~h}$, when the expression levels of GCase protein were relatively low (Supplementary Figures $5 \mathrm{a}$ and f). GlcCer levels were elevated by RSV, reaching a 6.4-fold increase over basal levels (Figure 6d). Sphingomyelin levels were also slightly increased (1.37-fold, Supplementary Figure S5g), consistent with a possible stimulatory role of RSV on de novo sphingolipid synthesis, as previously reported. ${ }^{33}$ However, the fold increase was significantly lower than that of GlcCer, suggesting that the latter may serve as a source for ceramide, sphingosine and S1P production via elevated GCase levels.

KD of GBA 1 by siRNA ablated the RSV induction of GCase protein and enzymatic activity (Figures $7 a$ and b). Importantly, RSV-induced autophagic cell death was attenuated by GBA1 $\mathrm{KD}$ (Figure 7c), consistent with the results from the original screen. In addition, GBA1 KD blocked the strong increase in LC3 lipidation levels detected at $48 \mathrm{~h}$ RSV treatment compared with non-targeting (NT) KD (Figure 7b). Sphingolipid profile analysis by mass spectrometry showed that RSV-induced increases in ceramide, specifically C16:0, 
C18:0, C20:0 and C24:1 acyl chains species, sphingosine and S1P were attenuated upon GBA1 KD (Figures $8 \mathrm{a}-\mathrm{c}$ and e). These results correlate the changes in sphingolipid profile with LC3B lipidation and cell death and indicate that GCase activity levels are critical for these lipid changes. Notably, no significant increase in GlcCer levels were observed upon the
KD of GBA1, either in untreated or RSV-treated cells (Figure $8 d$ ), similar to previous documentation in $\mathrm{GBA}(+/-$ ) mouse brains that displayed reduced GCase activity. ${ }^{34,35}$

Finally, ultrastructural analysis indicated a significant decrease in cytoplasmic AV area in RSV-treated GBA1 KD cells compared with NT KD cells (Figure 7d). Thus the a

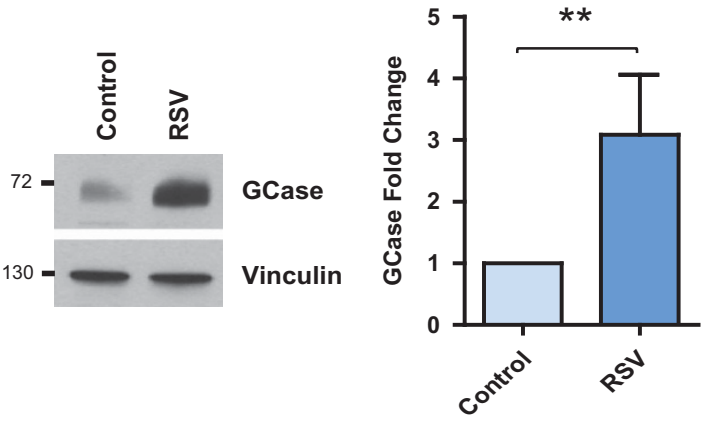

C

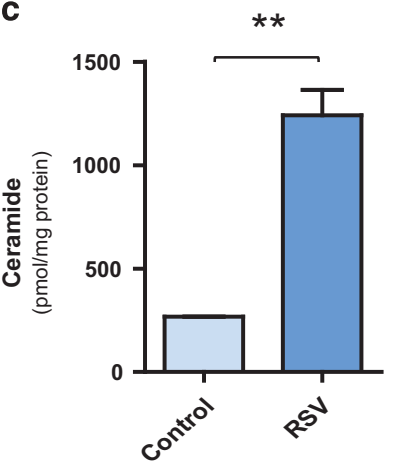

e

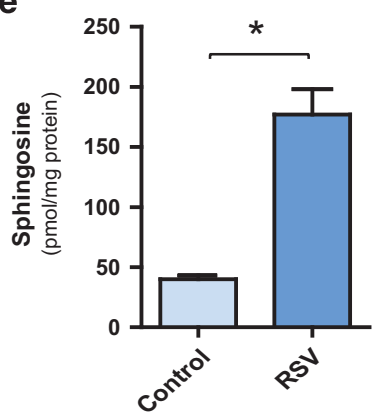

b

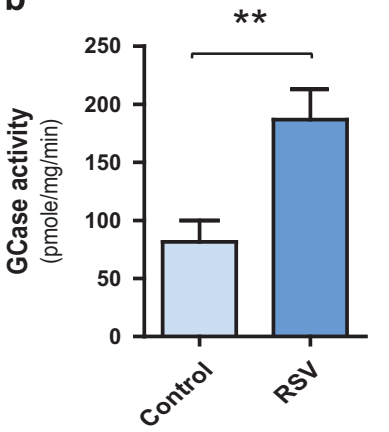

d

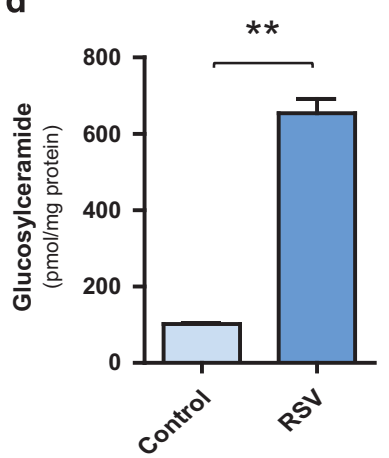

f

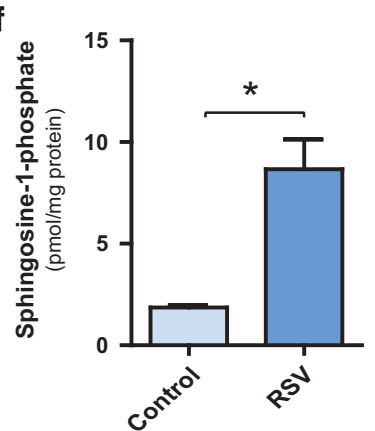

Figure 6 RSV induces autophagic cell death through GCase-regulated sphingolipid metabolism. (a) Western blotting of GCase in untreated and RSV (200 $\mu$ M; $48 \mathrm{~h}$ ) treated A549 cells (left) and quantitation by densitometry of GCase levels represented as mean \pm S.D. of four independent experiments, with untreated levels set at 1. (b) GCase activity measured in untreated and RSV $(200 \mu \mathrm{M} ; 48 \mathrm{~h})$ treated cells. Values are means \pm S.D. of three independent experiments. (c-f) Lipid mass spectrometric analysis of ceramide (c), GlcCer (d), sphingosine (e) and S1P (f) from untreated and RSV (200 $\mu \mathrm{M} ; 48 \mathrm{~h})$ treated A549 cells. Panels (c-f) represent mean \pm S.D. of two independent experiments; statistical significance determined by two-tailed unpaired Student's $t$-test, ${ }^{*} P<0.05 ;{ }^{* *} P<0.01$

Figure 7 GBA1 KD inhibits RSV-induced autophagic cell death. (a) GCase activity in siNT- and siGBA1-transfected cells treated with or without RSV (200 $\mu \mathrm{M}$; $48 \mathrm{~h}$ ). Data represents mean \pm S.D. of three replicate experiments; statistical significance was assessed using one-way analysis of variance (ANOVA) followed by Tukey's post hoc test, ${ }^{* \star *} P<0.001$. (b) Western blotting of GCase and LC3 lipidation in siNT- and siGBA1-transfected cells treated with or without RSV (200 $\left.\mu \mathrm{M} ; 48 \mathrm{~h}\right)$. (c) Cell viability was determined by CellTiter-Glo assay after $48 \mathrm{~h} \mathrm{RSV}$ treatment in siNT- and siGBA1-transfected A549 cells. Data are represented as a ratio of ATP levels in RSV-treated cells to untreated cells in siNT- and siGBA1-transfected A549 cells. Data represents mean \pm S.D. of six replicate experiments. Statistical significance determined by two-tailed unpaired Student's $t$-test, ${ }^{* * \star} P<0.0001$. (d) Quantitation of total cytoplasmic area covered by AVs ( $n=10$ cells) for siNTand siGBA1 cells untreated or treated with RSV (200 $\mu \mathrm{M}, 24 \mathrm{~h}$ ). (e) Representative images of siNT and siGBA1 KD cells treated with RSV for $24 \mathrm{~h}$. Data represent mean \pm S.D. of three replicate experiments. Statistical significance was assessed using one-way ANOVA followed by Tukey's post hoc test, ${ }^{* * *} P<0.001$ 
a

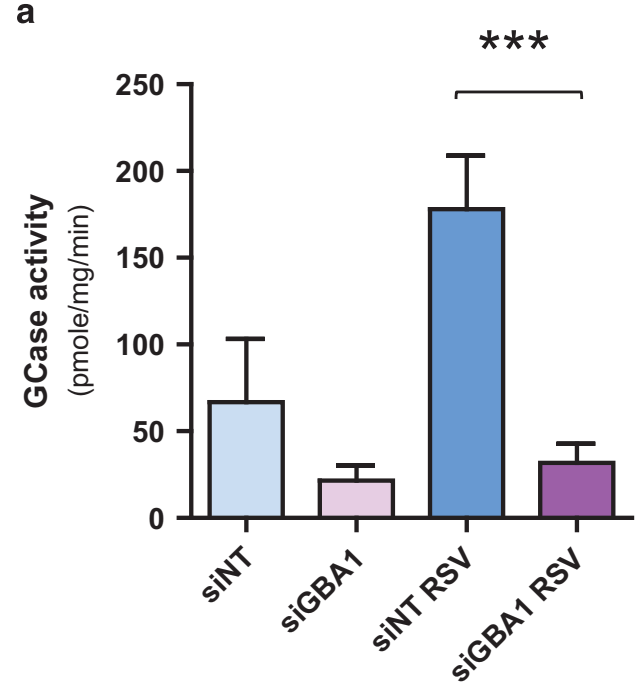

c

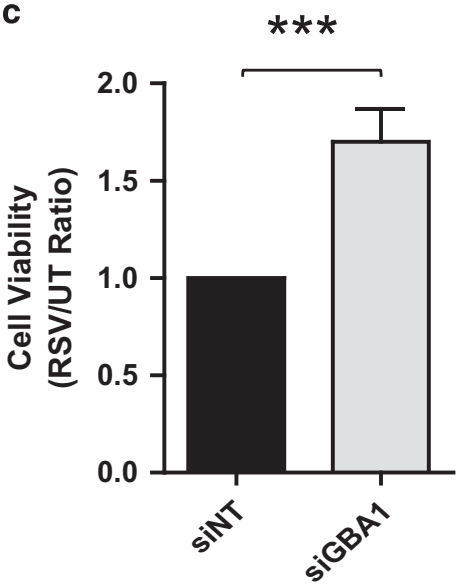

b

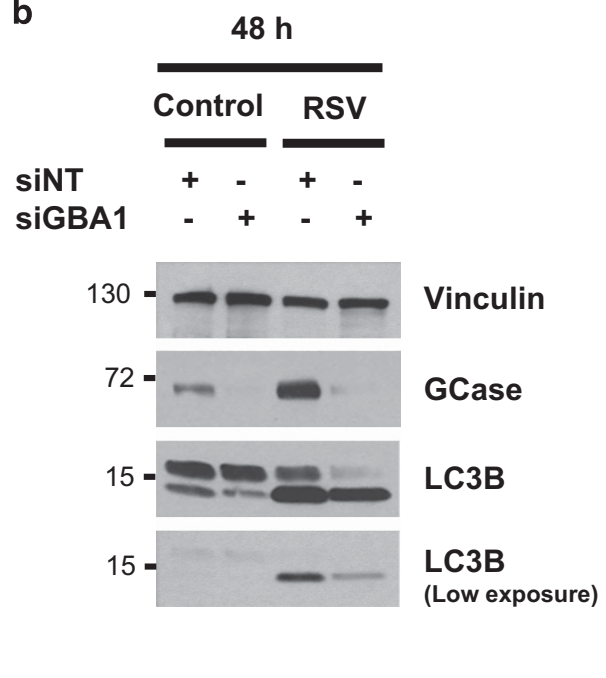

d

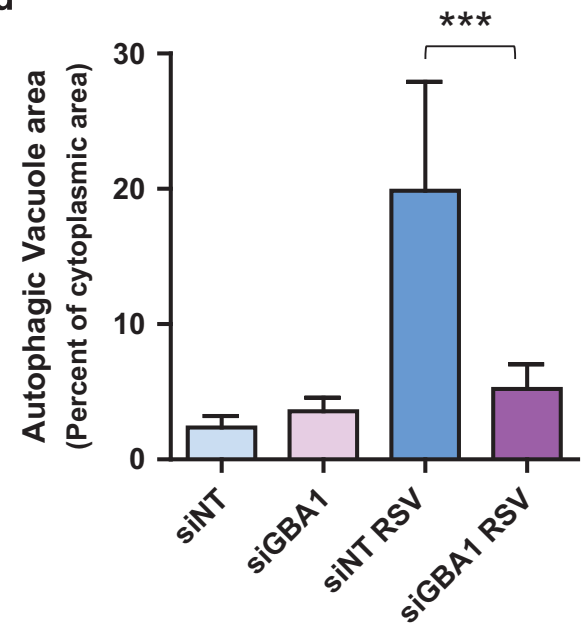

e

RSV

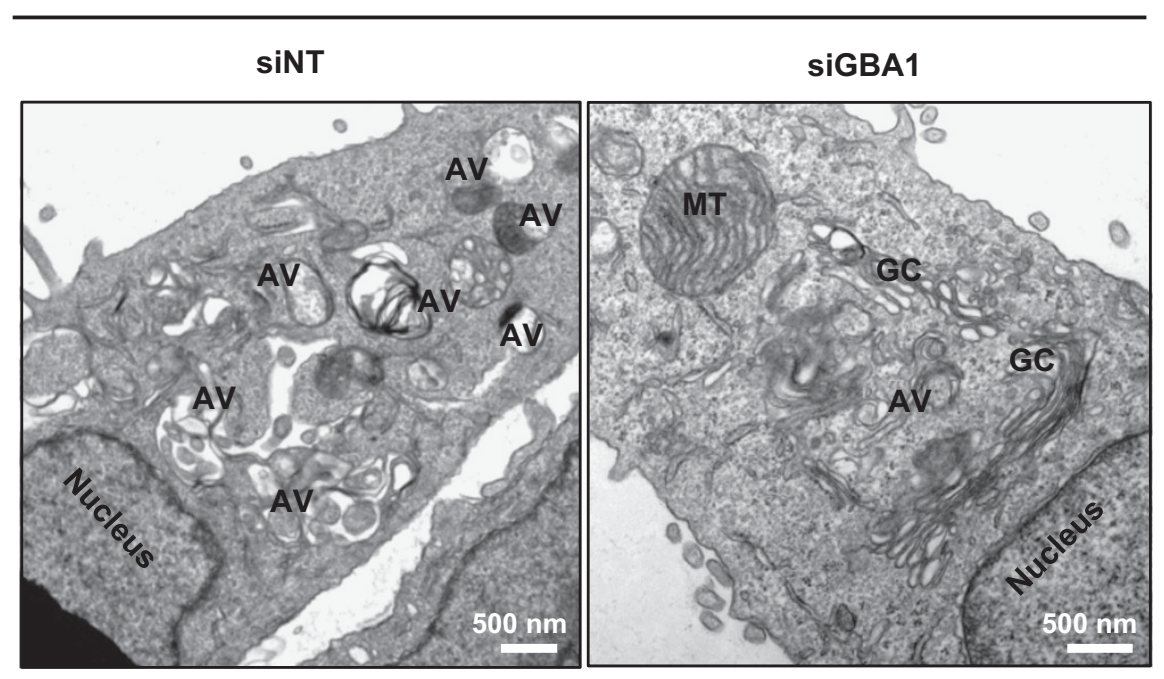


a

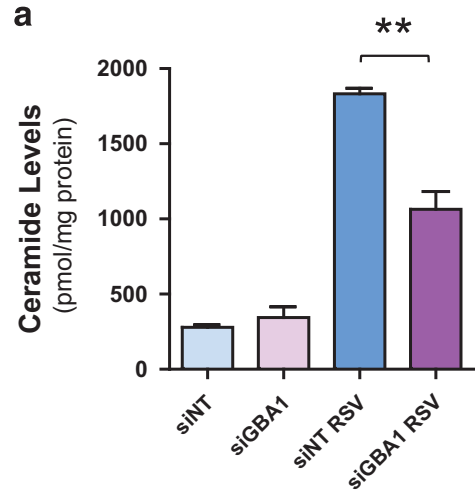

C

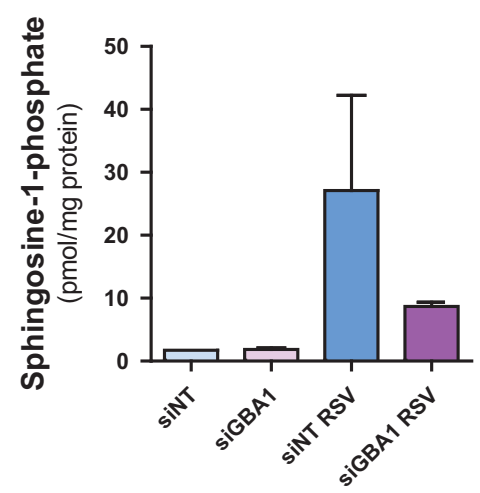

b

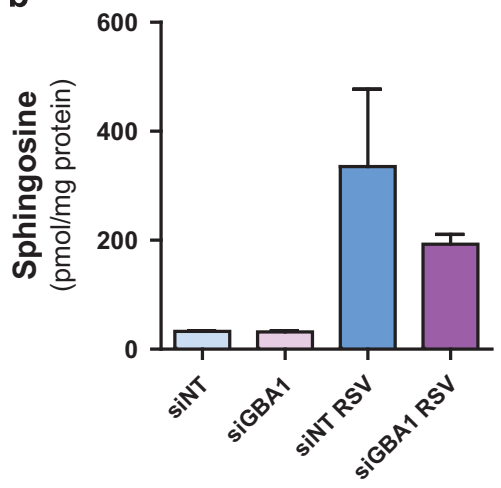

d

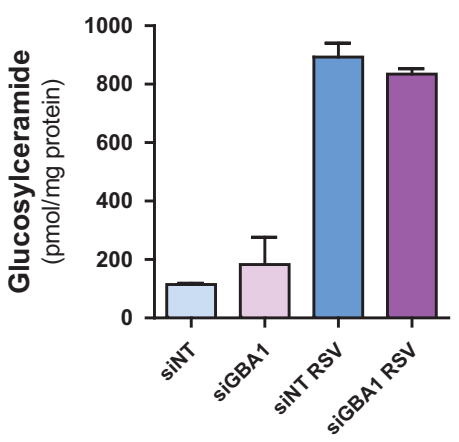

e

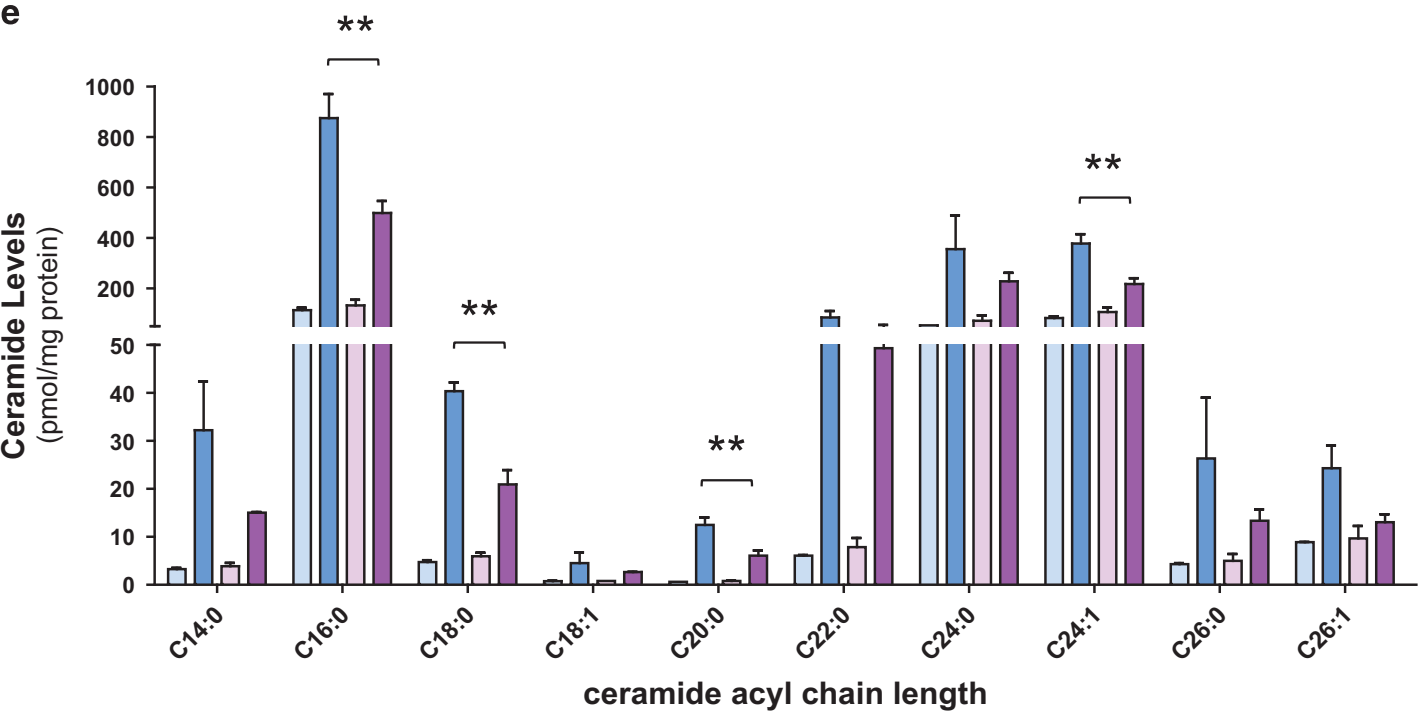

Figure 8 GBA1 KD changes sphingolpid levels. (a-e) Lipid mass spectrometric analysis of ceramide (a), sphingosine (b), S1P (c), GlcCer (d) and different chain length ceramide species (e) in siNT- and siGBA-transfected cells treated with and without RSV (200 $\mu \mathrm{M} ; 48 \mathrm{~h})$. Data represent mean \pm S.D. of two replicate experiments. Statistical significance was assessed using one-way analysis of variance followed by Tukey's post hoc test, ${ }^{* \star} P<0.01$

increased GCase activity in response to RSV contributes to the observed increase in LC3B lipidation and the resulting abundance of AVs. Importantly, ultrastructural morphology analysis showed that many of the RSV-induced hallmarks of autophagic cell death were alleviated by depletion of GBA1; RSV-treated GBA1 KD cells showed many normal mitochondria, Golgi and ER, compared with RSV-treated NT KD cells (Figure 7e). Taken together, these results prove the critical importance of increased GCase levels and the associated changes in sphingolipids in regulating autophagic cell death.

\section{Discussion}

In this work, we chose RSV-treated A549 cells as a model system for studying autophagic cell death, based on our findings that they showed a strong increase in autophagic flux 
and massive cell death, which did not involve apoptosis nor necroptosis but required the autophagy genes Atg4B, Atg7, Atg12 and MAP1LC3B. Unlike autosis, wherein cell death was not dependent on the late degradative stages within the autolysosome, ${ }^{22}$ massive degradation by the autolysosome persisted in this system until cell death was evident. Moreover, ultrastructural analysis indicated that the AVs occupied at least $25 \%$ of cytoplasmic area; this is likely an underestimate as this quantification did not include the empty vacuoles that were proven to be late-stage autolysosomes by immunogold labeling. Such profound autophagic activity is consistent with a self-consumption mechanism leading to cell dysfunction. In addition, endomembrane failure was observed. It is not clear if this results from depletion of membrane sources due to their re-routing to support the substantial autophagosome biogenesis or from autophagosome-dependent consumption of cellular membranes. The phenotype was further distinct from the previously described autosis in that membrane-bound electron-dense structures were not observed in the perinuclear space and plasma membrane injury was not prominent. ${ }^{22,36}$ Altogether, this work provides for the first time a detailed ultrastructural characterization of cells dying by self-consumption via strong continuous autophagic flux.

The Beclin-1- and Ulk1-independent nature of the autophagy activation, and its reliance on Golgi, suggests that RSV induced non-canonical autophagy-dependent cell death, consistent with previous findings. ${ }^{37}$ Non-canonical autophagy involving activation of the ubiquitin-like conjugation pathways and de novo autophagosome synthesis without the input of upstream signals from mTOR, Ulk1 and Vps34 has been previously described. ${ }^{25,26}$ Obviously, these systems bypass the previously reported feedback mechanisms in canonical autophagy that restrain self-consumption, such as reactivating mTOR, inhibiting $\mathrm{AMPK}^{38}$ or degrading ULK1, Beclin-1 and Vps34. ${ }^{39,40}$ Whether non-canonical autophagy systems use other types of brakes should be further investigated. Yet our work shows that, during autophagic cell death, a continuous unperturbed increase in the autophagic flux up to the terminal stages is essential.

To determine whether, in analogy to Dictyostelium, ${ }^{18,41,42}$ the sustained autophagic flux functions in tandem with other induced molecular pathways that together convert survival autophagy into autophagic cell death, we applied here for the first time an unbiased functional shRNA screen for discovering essential genes that mediate autophagic cell death. Our signalome-wide screen led to the identification of the lysosomal enzyme GCase, encoded by the GBA1 gene, as a significant hit that was validated in a secondary siRNA screen. RSV-induced autophagic cell death was accompanied by an increase in GCase expression and activity and accumulation of ceramide at late time points when the ultrastructural hallmarks of cell death were prominent. Most importantly, GBA1 KD decreased LC3B lipidation and rescued from the abnormal cellular ultrastructure changes and cell death. This prominent outcome of GBA1 KD indicates that the GCase-mediated pathway is rate limiting for driving the system toward autophagic cell death.

The direct targets of GCase-derived ceramides involved in the increased LC3 lipidation still need to be identified. C18-ceramide on mitochondrial membranes was shown to act as a receptor for LC3-II on autophagosomes or autolysosomes, leading to lethal mitophagy. ${ }^{43}$ Yet, in our system the self-digestion rescued by GBA1 KD is not restricted to mitochondria. Previous reports on ceramide-mediated signaling pathways such as activation of Beclin- 1 by JNK-mediated phosphorylation of its inhibitor, $\mathrm{Bcl}-2,{ }^{44}$ or by upregulation of Beclin-1 expression ${ }^{45}$ are obviously not relevant in our system. Intriguingly, besides a potential role in signaling, the sphingolipid content of membranes can affect their fusion capabilities and curvature, ${ }^{46}$ raising the hypothesis that the GCasemediated changes in sphingolipids may influence other steps in autophagosome/autolysosome formation, in addition to the enhancement of LC3 lipidation. Notably, previous reports indicate that ceramides can also trigger apoptosis in various cancer cell lines by forming channels in the mitochondrial membrane leading to caspase-3 activation. ${ }^{47,48}$ However, RSV has been shown to induce dihydroceramides in some cells, ${ }^{49}$ which can inhibit ceramide channel formation and apoptosis induction. ${ }^{50}$ Furthermore, the early and massive consumption of mitochondria by the enhanced autophagic flux induced by RSV may prevent activation of mitochondria-based apoptosis. Thus the final cellular outcome depends on the overall cell signaling milieu induced by the initial trigger, including the types of ceramides produced and their intracellular localization.

GCase has currently received significant attention as GBA1 loss-of-function mutations, which lead to Gaucher disease, are also a major risk factor for developing Parkinson disease. ${ }^{51}$ The latter connection has been attributed to decreased basal autophagic activity upon GBA1 inactivation, leading to accumulation of $a$-synuclein. ${ }^{52}$ In our study, in contrast to Gaucher disease, GBA1-mediated autophagy is detrimental due to its overactivation above basal levels. Thus the concept that loss of function and hyperactivation of GCase can both alter subcellular ceramide levels leading to misregulation of autophagy, with deleterious consequences for the cell, places $G B A 1$ in a critical rate-limiting junction.

To conclude, our data advance the field of autophagic cell death by providing a detailed ultrastructural characterization of the dying cells and a mechanistic insight that highlights two critical events: a continuous activation of autophagic flux over time with no brakes, and the induction of GCase expression and its enzymatic activity, which drives the system toward over self-consumption and membrane catastrophe.

\section{Materials and Methods}

Chemicals, cell culture and transfections. RSV and cyclohexamide (SigmaAldrich, St. Louis, MO, USA), BafA1 (LC Laboratories, Woburn, MA, USA), Q-VD-OPH (BioVision, Milpitas, CA, USA), LysoTracker Red DND-99 (Invitrogen, Carlsbad, CA, USA), $\mathrm{H}_{2} \mathrm{DCFDA}$ (Invitrogen), Hoechst 33342 (Invitrogen), NSA (EMD Millipore, Billerica, MA, USA), TRAIL (Peprotech, Rocky Hill, NJ, USA), NBD-C6-GluCer and NBD-C6-Cer (Avanti Polar Lipids, Alabaster, AL, USA) were obtained from the indicated suppliers. TNF (Ybdy, Seoul, South Korea), pan-caspase inhibitor z-VAD.fmk and bivalent IAP antagonist BV6 (Wuxi Apptec, Shanghai, China) were kindly provided by Professor David Wallach, Weizmann Institute of Science, Rehovot, Israel and Brefeldin A (Calbiochem, San Diego, CA, USA) by Dr. Yifat Merbl, Weizmann Institute of Science, Israel. Unless specified, all other chemicals and reagents were purchased from Sigma-Aldrich.

A549 lung adenocarcinoma cells and human HT29 colorectal cancer cells were obtained from ATCC (Manassas, VA, USA) and verified to be mycoplasma free by periodical PCR testing. They were maintained in DMEM and McCoy's $5 \mathrm{~A}$ medium (Biological Industries, Cromwell, CT, USA), respectively. Both media were supplemented with $2 \mathrm{mM}$ glutamine (Gibco, Saint Aubin, France), $100 \mathrm{U} / \mathrm{ml}$ penicillin and streptomycin (Gibco) and 10\% FBS (Hyclone, Logan, UT, USA). Transient transfections of siRNA and DNA were performed using Lipofectamine2000 
(Invitrogen) as per the manufacturer's instructions. siRNA validation screen was performed in a 96-well format in reverse-transfection mode using DharmaFect1 transfection reagent (Dharmacon, Lafayette, CO, USA).

Clonogenic survival and cell viability assays. A549 cells untreated or treated with $200 \mu \mathrm{M}$ RSV for $48 \mathrm{~h}$ were washed, trypsinized, counted and plated in 12-well plates. Cells were allowed to grow and form colonies for 1 week, followed by staining with a mixture of $6.0 \%$ glutaraldehyde and $0.5 \%$ crystal violet for $30 \mathrm{~min}$. The plates were then rinsed with water and left to dry at room temperature. Plates with colonies were scanned using HP Scanner (HPE, Ra'anana, Israel).

Cell viability was measured using luminescence based CellTiter-Glo assay according to the manufacturer's instructions (Promega, Madison, WI, USA). Luminescence was read in a Veritas microplate luminometer (Turner BioSystems, Sunnyvale, CA, USA). In siRNA KD experiments, cell viability was calculated by dividing luminescence from RSVtreated wells by their corresponding vehicle treatment wells, which was further normalized to their respective siNT (Non-Targeting) negative control.

Live cell staining and fluorescence microscopy. A549 cells transfected with RFP-GFP-LC3 construct (a kind gift from Professor Tamotsu Yoshimori, Osaka University, Japan) or co-transfected with GFP-LC3 and mCherryGalT (a kind gift from Professor Orly Reiner, Weizmann Institute of Science, Rehovot, Israel) were plated onto poly-L-Lysine (Sigma-Aldrich) coated $13 \mathrm{~mm}$ glass coverslips. After attachment, cells were treated with $200 \mu \mathrm{M}$ RSV (Sigma Aldrich) for an additional $24 \mathrm{~h}$. Cells were fixed in $3.7 \%$ paraformaldehyde and viewed by fluorescence microscopy (Olympus BX41, Olympus, Tokyo, Japan) with $60 \times$ (NA 1.25) UPlan-Fl oil immersion objective. Control and RSV $(200 \mu \mathrm{M} ; 48 \mathrm{~h})$ treated cells were incubated with LysoTracker Red DND-99 (Invitrogen) at a final concentration of $100 \mathrm{nM}$ and imaged by fluorescence microscopy. Approximately 200 cells per sample were counted for quantification of pattern of LysoTracker Red staining in triplicate experiments. Cell death by apoptosis was determined by Hoechst 33342 nuclear fragmentation assay as previously described. ${ }^{53}$ Briefly, cells treated with or without RSV $(200 \mu \mathrm{M} ; 24$ and $48 \mathrm{~h})$ and $100 \mathrm{ng} / \mathrm{ml}$ TRAlL with $20 \mu \mathrm{g} / \mathrm{ml} \mathrm{CHX}$ were incubated in $100 \mathrm{ng} / \mathrm{ml}$ Hoechst 33342 for $15 \mathrm{~min}$ in dark followed by rinsing in PBS and imaged by fluorescence microscopy. Control and RSV $(200 \mu \mathrm{M} ; 48 \mathrm{~h})$ treated cells were incubated with ROS detector $\mathrm{H}_{2}$ DCFDA at a final concentration of $10 \mu \mathrm{M}$ for $30 \mathrm{~min}$, washed and imaged by fluorescence microscopy. Digital images were obtained using DP50 CCD camera with the ViewfinderLite and StudioLite software (Olympus, Tokyo, Japan). A549 cells transfected with LAMP1-GFP (Addgene, Cambridge, MA, USA) or RFP-GFP-LC3 constructs in parallel were treated with or without RSV $24 \mathrm{~h}$, followed by fixation for immunoelectron microscopy.

TEM, immunogold labeling, and morphometric analysis. A549 cells treated with or without RSV for 8,24 and $48 \mathrm{~h}$ were fixed with $3 \%$ paraformaldehyde, $2 \%$ glutaraldehyde in $0.1 \mathrm{M}$ cacodylate buffer containing $5 \mathrm{mM}$ $\mathrm{CaCl}_{2}$ (pH 7.4), followed by $1 \%$ osmium tetroxide supplemented with $0.5 \%$ potassium hexacyanoferrate tryhidrate and potasssium dichromate in $0.1 \mathrm{M}$ cacodylate $(1 \mathrm{~h})$, stained with $2 \%$ uranyl acetate in water $(1 \mathrm{~h})$, dehydrated in graded ethanol solutions and embedded in Agar 100 epoxy resin (Agar Scientific Ltd., Stansted, Essex, UK). Ultrathin sections (70-90 nm) were cut and processed for imaging. Immunogold labeling was performed in cells fixed in $4 \%$ paraformaldehyde with $0.1 \%$ glutaraldehyde in $0.1 \mathrm{M}$ cacodylate buffer $(\mathrm{pH}=7.4)$ for $1 \mathrm{~h}$ at room temperature. Fixed cells were pelleted and embedded in gelatin, soaked overnight in $2.3 \mathrm{M}$ sucrose and rapidly frozen under liquid nitrogen. Frozen ultrathin $(70-90 \mathrm{~nm})$ sections were cut with a diamond knife at $-120^{\circ} \mathrm{C}$ on a Leica EM UC6 ultramicrotome (Leica Microsystems GmbH, Wetzlar, Germany). The sections were collected on 200-mesh Formvar-coated nickel grids. Sections were blocked with a solution containing $1 \% \mathrm{BSA}, 0.1 \%$ glycine, $0.1 \%$ gelatin and $1 \%$ Tween 20. Immuno-labeling was performed using affinity-purified anti-GFP antibodies $\left(1: 50\right.$, Abcam, Cambridge, MA, USA), overnight at $4{ }^{\circ} \mathrm{C}$, followed by exposure to goat anti-Rabbit IgG coupled to 10 -nm gold particles $(1: 20$, Jackson ImmunoResearch, West Grove, PA, USA), for $30 \mathrm{~min}$ at room temperature. Contrast staining and embedding were performed as previously described. ${ }^{54}$ The sections were viewed and photographed with a FEI Tecnai SPIRIT (FEI, Eindhoven, The Netherlands) transmission electron microscope operated at $120 \mathrm{kV}$ and equipped with an EAGLE CCD Camera (FEl, Eindhoven, The Netherlands).

Morphometric measurements were performed on digital micrograph images using the Image J software (FEl, Eindhoven, The Netherlands). The number of AVs per cell area was quantified by double blind analysis. AV area and cytoplasmic areas edges were traced and density was determined. The amount of cytoplasmic area occupied by AVs was determined by dividing the observed densities of AV area by cytoplasmic area of the cell.

Immunoblotting. Cells were lysed in PLB buffer ( $0.1 \mathrm{M}$ sodium phosphate, $\mathrm{pH}$ 7.5, $100 \mathrm{mM} \mathrm{NaCl}, 0.1 \%$ SDS, $1 \%$ Triton X-100, $1 \%$ sodium deoxycholate) supplemented with $1 \%$ protease inhibitors and $1 \mathrm{mM}$ PMSF. In experiments in which phosphorylated proteins were analyzed, $1 \mathrm{mM}$ sodium orthovanadate was added to the lysis buffer. Proteins separated on SDS-PAGE were blotted onto nitrocellulose membranes, which were blocked and incubated with antibodies for LC3B, ATG7 and GBA1 (Sigma-Aldrich), cleaved caspase-3, caspase-8 and cleaved caspase-9 (Cell Signaling, Danvers, MA, USA), p62 (BD Biosciences, San Jose, CA, USA), Atg12 (MBL Life Science, Woburn, MA, USA), Beclin 1 (SantaCruz Biotech, Santa Cruz, CA, USA), ULK1 (Abcam). Total MLKL (Gene Tex, Irvine, CA, USA) and phospho MLKL (S358) antibody (Abcam) were a kind gift from Professor David Wallach, Weizmann Institute of Science, Rehovot, Israel. Detection was performed with goat anti-mouse or goat anti-rabbit HRP-conjugated secondary antibodies (Jackson ImmunoResearch), followed by chemiluminescence using EZ-ECL Solution (Biological Industries).

Primary shRNA screen. A549 cells were transduced with DECIPHER $27 \mathrm{~K}$ Pooled shRNA library-Human Module 1 (Cellecta, Mountain View, CA, USA). The library consists of 27500 shRNAs targeting 5043 genes (approximately five or six shRNAs per gene) in the pRSI12-U6-(sh)-HTS4-UbiC-TagRFP-2A-Puro expression vector. In the pRSI12 expression vector, the shRNA is expressed under wild-type U6 RNA polymerase III promoter and TagRFP (Evrogen, Moscow, Russia) and puromycin resistance genes under human ubiquitin $C$ promoter. In addition, all shRNAs have unique, optimized 18-nt barcode sequences, which facilitate HighThroughput Sequencing data analysis and identification of functional shRNAs. Seventy million cells were transduced with the shRNA library at $\mathrm{MOI}$ of 0.3 (allowing each individual shRNA of the library with the complexity of 27500 to be delivered to at least 700 cells). After $48 \mathrm{~h}$, infected cells were selected with puromycin $(2 \mu \mathrm{g} / \mathrm{ml})$ for an additional $48 \mathrm{~h}$. On completion of puromycin treatment, cells were washed, trypsinized, counted and split into two populations. The first population was snap frozen on dry ice and stored at $-80^{\circ} \mathrm{C}$ refrigerator as TO reference time point. The second population was treated with $200 \mu \mathrm{M}$ RSV for 4 days. After RSV treatment, surviving cells were seeded at optimal density (5 million cells per $15 \mathrm{~cm}$ plate) and allowed to proliferate for another 4 days. Later, the cells were pelleted, snap-frozen and stored at $-80^{\circ} \mathrm{C}$ refrigerator as T8 time point. The frozen samples (T0 and T8) were submitted to Cellecta, Inc. for genomic DNA extraction, bar code amplification, high-throughput sequencing and deconvolution analysis.

Data analysis was performed for all samples by normalizing each individual sample to 20 million reads, followed by dividing it by the mean of the shRNAs targeting Luciferase gene. At individual shRNA level, Student's $t$-test for each shRNA was performed and genes with three or more shRNAs showing a significant abundance (fold change $\geq 1.5$ or $\log _{2}$ fold change $\geq 0.58 ; P \leq 0.05$ and FDR $<0.1$ ) were considered as positive hits. Fifty five genes that passed the set criterion as mentioned above were passed through a sum of ranked standardization score algorithm ${ }^{31}$ to rank the hits. The genes were ranked based on gene scores obtained by the formula: $\mu /(\sigma / \sqrt{ } n)$, where $\mu$ is the mean $\log _{2}$ fold-change ratios of shRNAs targeting the gene of interest, $\sigma$ denotes the variance of the $\log _{2}$ fold-change ratios and $n$ corresponds to number of shRNAs targeting the gene. The top 24 hits emerging from this analysis were taken forward for the secondary siRNA-based validation screen.

siRNA validation screen. A549 cells were reverse transfected in 96-well plates with $50 \mathrm{nM}$ indicated siGENOME siRNA pools (Dharmacon) using DharmaFECT1 transfection reagent (Dharmacon). Cells were treated $48 \mathrm{~h}$ posttransfection with $200 \mu \mathrm{M}$ RSV for an additional $48 \mathrm{~h}$. Cell viability was measured using CellTiter-Glo assay kit (Promega), as described above. siRNA targeting MAPLC3B (Dharmacon) was used as a positive control, while nontargeting pool 2, RISC-free siRNA, non-targeting 5 (NT5) and Mock transfection served as negative controls. An identical protocol was used for siGBA1 validation experiments using ON-TARGETplus siRNA pools (Dharmacon). For three independent biological replicates of the siRNA validation screen, relative luminescence units was obtained by normalizing the RSV-treated sample plate with the corresponding vehicle-treated plate. The ratio of RSV-treated wells to their corresponding vehicle-treated wells was calculated for all the wells, and the data were further normalized to the respective RISC-free siRNA negative control. 
Positive hits were defined as genes whose $\mathrm{KD}$ led to a significant increase (fold-change $1.5 ; P \leq 0.05$ ) in cell viability in comparison to the corresponding RISC-free siRNA control.

GCase activity. GCase was assayed as described ${ }^{55}$ with some modifications. Briefly, $8 \mu \mathrm{M}$ C6-NBD-GlcCer was incubated with $5 \mu \mathrm{g}$ of a cell lysate in a final volume of $250 \mu \mathrm{l}$ Mcllvaine's buffer $(\mathrm{pH} 4)$ for $1 \mathrm{~h}$ at $37^{\circ} \mathrm{C}$. The reactions were terminated by addition of chloroform:methanol $\left(1: 2\right.$, vol:vol), lipids extracted ${ }^{56}$ and separated by thin layer chromatography using chloroform:methanol: $9.8 \mathrm{mM} \mathrm{CaCl}_{2}$ $(60: 35: 8$, vol:vol:vol) as the developing solvent. NBD-GlcCer and NBD-ceramide were imaged using Typhoon 9410 Variable Mode Imager and bands quantified using Image-QuantTL (GE Healthcare, Rehovot, Israel).

Sphingolipid analysis. Untransfected and siRNA-transfected A549 cells treated with or without RSV were snap frozen under liquid nitrogen and dried by lyophilization. LC-ESI-MS/MS was performed using an $A B I 4000$ quadrupole linear

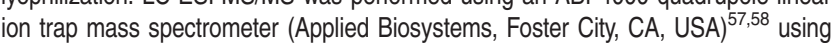
sphingolipid internal standards from Avanti Polar Lipids.

Statistical analysis. The statistical significance of differences between means was assessed either by standard Student's t-test or by one-way ANOVA followed by Tukey's post hoc test, as mentioned in the figure legends. Values with $P<0.05$ were considered significant.

\section{Conflict of Interest}

The authors declare no conflict of interest.

Acknowledgements. We thank Einat Vitner for helpful discussions and Ester Feldmesser for help with statistics. We thank Helena Sabanay, Chen Yaacobi and Samuel L. Kelly for technical assistance. This work was supported by a grant from the European Research Council under the European Union's Seventh Framework Program (FP7/2007-2013/ERC grant agreement 322709). AHF is the Joseph Meyerhoff Professor of Biochemistry and AK is the incumbent of the Helena Rubinstein Chair of Cancer Research.

1. Mizushima N, Komatsu M. Autophagy: renovation of cells and tissues. Cell 2011; 147: 728-741.

2. Young MM, Takahashi Y, Khan O, Park S, Hori T, Yun J et al. Autophagosomal membrane serves as platform for intracellular death-inducing signaling complex (iDISC)-mediated caspase-8 activation and apoptosis. J Biol Chem 2012; 287: 12455-12468.

3. Laussmann MA, Passante E, Dussmann H, Rauen JA, Wurstle ML, Delgado ME et al. Proteasome inhibition can induce an autophagy-dependent apical activation of caspase-8. Cell Death Differ 2011; 18: 1584-1597.

4. Pyo JO, Jang MH, Kwon YK, Lee HJ, Jun JI, Woo HN et al. Essential roles of Atg5 and FADD in autophagic cell death: dissection of autophagic cell death into vacuole formation and cell death. J Biol Chem 2005; 280: 20722-20729.

5. Jin Z, Li Y, Pitti R, Lawrence D, Pham VC, Lill JR et al. Cullin3-based polyubiquitination and p62-dependent aggregation of caspase-8 mediate extrinsic apoptosis signaling. Cell 2009; 137: $721-735$

6. Goodall ML, Fitzwalter BE, Zahedi S, Wu M, Rodriguez D, Mulcahy-Levy JM et al. The autophagy machinery controls cell death switching between apoptosis and necroptosis. Dev Cell 2016; 37: 337-349.

7. Yu L, Wan F, Dutta S, Welsh S, Liu Z, Freundt E et al. Autophagic programmed cell death by selective catalase degradation. Proc Natl Acad Sci USA 2006; 103: 4952-4957.

8. Basit $F$, Cristofanon $S$, Fulda S. Obatoclax (GX15-070) triggers necroptosis by promoting the assembly of the necrosome on autophagosomal membranes. Cell Death Differ 2013; 20 : 1161-1173.

9. Nezis IP, Shravage BV, Sagona AP, Lamark T, Bjorkoy G, Johansen T et al. Autophagic degradation of dBruce controls DNA fragmentation in nurse cells during late Drosophila melanogaster oogenesis. J Cell Biol 2010; 190: 523-531.

10. Gump JM, Staskiewicz L, Morgan MJ, Bamberg A, Riches DW, Thorburn A. Autophagy variation within a cell population determines cell fate through selective degradation of Fap-1. Nat Cell Biol 2014; 16: 47-54

11. He W, Wang Q, Srinivasan B, Xu J, Padilla MT, Li Z et al. A JNK-mediated autophagy pathway that triggers c-IAP degradation and necroptosis for anticancer chemotherapy. Oncogene 2014; 33: 3004-3013

12. Hou W, Xie Y, Song X, Sun X, Lotze MT, Zeh HJ 3rd et al. Autophagy promotes ferroptosis by degradation of ferritin. Autophagy 2016; 12: 1425-1428.

13. Lockshin RA, Zakeri Z. Apoptosis, autophagy, and more. Int J Biochem Cell Biol 2004; 36 : 2405-2419.
14. Clarke PG. Developmental cell death: morphological diversity and multiple mechanisms. Anat Embryol (Berl) 1990; 181: 195-213.

15. Zakeri Z, Bursch W, Tenniswood M, Lockshin RA. Cell death: programmed, apoptosis, necrosis, or other? Cell Death Differ 1995; 2: 87-96.

16. Denton D, Nicolson S, Kumar S. Cell death by autophagy: facts and apparent artefacts Cell Death Differ 2012; 19: 87-95.

17. Shen H-M, Codogno P. Autophagic cell death: Loch Ness monster or endangered species? Autophagy 2011; 7: 457-465.

18. Giusti C, Luciani MF, Golstein P. A second signal for autophagic cell death? Autophagy 2010; 6: 823-824.

19. Berry DL, Baehrecke EH. Growth arrest and autophagy are required for salivary gland cell degradation in Drosophila. Cell 2007; 131: 1137-1148.

20. Denton D, Shravage B, Simin R, Mills K, Berry DL, Baehrecke EH et al. Autophagy, not apoptosis, is essential for midgut cell death in Drosophila. Curr Biol 2009; 19: 1741-1746.

21. Koike M, Shibata M, Tadakoshi M, Gotoh K, Komatsu M, Waguri S et al. Inhibition of autophagy prevents hippocampal pyramidal neuron death after hypoxic-ischemic injury. $A m$ J Pathol 2008; 172: 454-469.

22. Liu Y, Shoji-Kawata S, Sumpter RM Jr., Wei $Y$, Ginet V, Zhang $L$ et al. Autosis is a Na+,K +-ATPase-regulated form of cell death triggered by autophagy-inducing peptides, starvation, and hypoxia-ischemia. Proc Natl Acad Sci USA 2013; 110: 20364-20371.

23. Kheloufi M, Boulanger $\mathrm{CM}$, Codogno $\mathrm{P}$, Rautou $\mathrm{PE}$. Autosis occurs in the liver of patients with severe anorexia nervosa. Hepatology 2015; 62: 657-658.

24. Yoon S, Bogdanov K, Kovalenko A, Wallach D. Necroptosis is preceded by nuclear translocation of the signaling proteins that induce it. Cell Death Differ 2016; 23: 253-260.

25. Gao Y, Liu Y, Hong L, Yang Z, Cai X, Chen X et al. Golgi-associated LC3 lipidation requires V-ATPase in noncanonical autophagy. Cell Death Dis 2016; 7: e2330.

26. Niso-Santano M, Malik SA, Pietrocola F, Bravo-San Pedro JM, Marino G, Cianfanelli V et al. Unsaturated fatty acids induce non-canonical autophagy. EMBO J 2015; 34: 1025-1041.

27. de Santi C, Pietrabissa A, Mosca F, Pacifici GM. Glucuronidation of resveratrol, a natural product present in grape and wine, in the human liver. Xenobiotica 2000; 30 1047-1054.

28. Brill SS, Furimsky AM, Ho MN, Furniss MJ, Li Y, Green AG et al. Glucuronidation of transresveratrol by human liver and intestinal microsomes and UGT isoforms. I Pharm Pharmacol 2006; 58: 469-479.

29. Gao M, Monian P, Quadri N, Ramasamy R, Jiang X. Glutaminolysis and transferrin regulate ferroptosis. Mol Cell 2015; 59: 298-308.

30. Gao M, Monian P, Pan Q, Zhang W, Xiang J, Jiang X. Ferroptosis is an autophagic cell death process. Cell Res 2016; 26: 1021-1032.

31. Sroczynska P, Cruickshank V, Bukowski J, Miyagi S, Bagger F, Walfridsson J et al. shRNA screening identifies JMJD1C as being required for leukemia maintenance. Blood 2014; 123: 1870-1882

32. Tidhar R, Futerman AH. The complexity of sphingolipid biosynthesis in the endoplasmic reticulum. Biochim Biophys Acta 2013; 1833: 2511-2518.

33. Scarlatti F, Sala G, Somenzi G, Signorelli P, Sacchi N, Ghidoni R. Resveratrol induces growth inhibition and apoptosis in metastatic breast cancer cells via de novo ceramide signaling. FASEB J 2003; 17: 2339-2341.

34. Sardi SP, Clarke J, Kinnecom C, Tamsett TJ, Li L, Stanek LM et al. CNS expression of glucocerebrosidase corrects alpha-synuclein pathology and memory in a mouse model of Gaucher-related synucleinopathy. Proc Natl Acad Sci USA 2011; 108: 12101-12106.

35. Enquist IB, Lo Bianco C, Ooka A, Nilsson E, Mansson JE, Ehinger M et al. Murine models of acute neuronopathic Gaucher disease. Proc Natl Acad Sci USA 2007; 104: 17483-17488.

36. Liu Y, Levine B. Autosis and autophagic cell death: the dark side of autophagy. Cell Death Differ 2015; 22: 367-376.

37. Mauthe M, Jacob A, Freiberger S, Hentschel K, Stierhof YD, Codogno P et al. Resveratrolmediated autophagy requires WIPI-1-regulated LC3 lipidation in the absence of induced phagophore formation. Autophagy 2011; 7: 1448-1461.

38. Alers S, Loffler AS, Wesselborg S, Stork B. Role of AMPK-mTOR-Ulk1/2 in the regulation of autophagy: cross talk, shortcuts, and feedbacks. Mol Cell Biol 2012; 32: 2-11.

39. Liu CC, Lin YC, Chen YH, Chen CM, Pang LY, Chen HA et al. Cul3-KLHL20 ubiquitin ligase governs the turnover of ULK1 and VPS34 complexes to control autophagy termination. Mol Cell 2016; 61: 84-97.

40. Feng Y, Klionsky DJ. Downregulation of autophagy through CUL3-KLHL20-mediated turnover of the ULK1 and PIK3C3/VPS34 complexes. Autophagy 2016; 12: 1071-1072.

41. Luciani MF, Kubohara Y, Kikuchi H, Oshima Y, Golstein P. Autophagic or necrotic cell death triggered by distinct motifs of the differentiation factor DIF-1. Cell Death Differ 2009; 16 : 564-570

42. Song Y, Luciani MF, Giusti C, Golstein P. c-di-GMP induction of Dictyostelium cell death requires the polyketide DIF-1. Mol Biol Cell 2015; 26: 651-658.

43. Sentelle RD, Senkal CE, Jiang W, Ponnusamy S, Gencer S, Selvam SP et al. Ceramide targets autophagosomes to mitochondria and induces lethal mitophagy. Nat Chem Biol 2012; 8: 831-838.

44. Pattingre S, Bauvy C, Carpentier S, Levade T, Levine B, Codogno P. Role of JNK1-dependent Bcl-2 phosphorylation in ceramide-induced macroautophagy. J Biol Chem 2009; 284: 2719-2728.

45. Scarlatti F, Bauvy C, Ventruti A, Sala G, Cluzeaud F, Vandewalle A et al. Ceramide-mediated macroautophagy involves inhibition of protein kinase $B$ and up-regulation of beclin 1. J Biol Chem 2004; 279: 18384-18391. 
46. Harvald EB, Olsen AS, Faergeman NJ. Autophagy in the light of sphingolipid metabolism. Apoptosis 2015; 20: 658-670.

47. Obeid LM, Linardic CM, Karolak LA, Hannun YA. Programmed cell death induced by ceramide. Science 1993; 259: 1769-1771.

48. von Haefen C, Wieder T, Gillissen B, Starck L, Graupner V, Dorken B et al. Ceramide induces mitochondrial activation and apoptosis via a Bax-dependent pathway in human carcinoma cells. Oncogene 2002; 21: 4009-4019.

49. Signorelli P, Munoz-Olaya JM, Gagliostro V, Casas J, Ghidoni R, Fabrias G. Dihydroceramide intracellular increase in response to resveratrol treatment mediates autophagy in gastric cancer cells. Cancer Lett 2009; 282: 238-243.

50. Stiban J, Fistere D, Colombini M. Dihydroceramide hinders ceramide channel formation: implications on apoptosis. Apoptosis 2006; 11: 773-780.

51. Futerman AH, Hardy J. Perspective: Finding common ground. Nature 2016; 537: S160-S161.

52. Du TT, Wang L, Duan CL, Lu LL, Zhang JL, Gao G et al. GBA deficiency promotes SNCA/ alpha-synuclein accumulation through autophagic inhibition by inactivated PPP2A. Autophagy 2015; 11: 1803-1820.

53. Crowley LC, Marfell BJ, Waterhouse NJ. Analyzing cell death by nuclear staining with Hoechst 33342. Cold Spring Harb Protoc 2016; 2016: pdb prot087205.

54. Tokuyasu KT. Application of cryoultramicrotomy to immunocytochemistry. J Microsc 1986; 143(Pt 2): 139-149.

55. Farfel-Becker T, Vitner EB, Kelly SL, Bame JR, Duan J, Shinder V et al. Neuronal accumulation of glucosylceramide in a mouse model of neuronopathic Gaucher disease leads to neurodegeneration. Hum Mol Genet 2014; 23: 843-854.
56. Bligh EG, Dyer WJ. A rapid method of total lipid extraction and purification. Can J Biochem Physiol 1959; 37: 911-917.

57. Sullards MC, Liu Y, Chen Y, Merrill AH Jr. Analysis of mammalian sphingolipids by liquid chromatography tandem mass spectrometry (LC-MS/MS) and tissue imaging mass spectrometry (TIMS). Biochim Biophys Acta 2011; 1811: 838-853.

58. Shaner RL, Allegood JC, Park H, Wang E, Kelly S, Haynes CA et al. Quantitative analysis of sphingolipids for lipidomics using triple quadrupole and quadrupole linear ion trap mass spectrometers. J Lipid Res 2009; 50: 1692-1707.

(c) (i) (s) $\odot$ This work is licensed under a Creative Commons Attribution-NonCommercial-NoDerivs 4.0 International License. The images or other third party material in this article are included in the article's Creative Commons license, unless indicated otherwise in the credit line; if the material is not included under the Creative Commons license, users will need to obtain permission from the license holder to reproduce the material. To view a copy of this license, visit http://creativecommons.org/licenses/by-nc-nd/4.0/

(C) The Author(s) 2017

Supplementary Information accompanies this paper on Cell Death and Differentiation website (http://www.nature.com/cdd) 